\title{
LA INVERSIÓN FIJA EN EL SECTOR MANUFACTURERO MEXICANO 1985-94: EL ROL DE LOS FACTORES FINANCIEROS Y EL IMPACTO DE LA LIBERALIZACIÓN FINANCIERA
}

\author{
Gaston Gelos \\ Alejandro Werner ${ }^{1}$ \\ Octubre de 1998 \\ Documento de Investigación No. 9805 \\ Dirección General de Investigación Económica \\ Banco de México
}

${ }^{1}$ Los autores son estudiante de doctorado en la Universidad de Yale e investigador de la Dirección de
Estudios Económicos del Banco de México, respectivamente. Gaston Gelos quisiera agradecer a Agustín
Carstens y a Moisés Schwartz por brindarle la oportunidad de trabajar sobre este tema como investigador
huésped en el Banco de México y por toda la ayuda prestada durante ese período. Dicho autor agradece la
amabilidad de Miguel Cervera del INEGI, y la oportunidad de realizar una parte importante de las
investigaciones en esa institución en Aguascalientes. También del INEGI, los autores agradecen
especialmente a Alejandro Cano, Abigail Durán, Eduardo Moreno, y a Ramón Sánchez por su ayuda.
Valiosos comentarios de Fernando Aportela, William Brainard, Sara Castellanos, Rafael Gamboa, Juan
Pablo Graf, Alfonso Guerra, Luis Mario Hernández, Michael Krause, Miguel Messmacher, Giuseppe
Moscarini, Mauricio Naranjo, y de Abraham Vela contribuyeron a mejorar la calidad del presente estudio.
Alexis Milo, Jorge Pastrana y Juan Pedro Treviño ayudaron en la obtención y preparación de datos.
Obviamente, ninguna de las personas mencionadas es responsable de los errores que pudieran encontrarse en
este trabajo. Las opiniones contenidas en este documento corresponden exclusivamente a los autores y no
representan el punto de vista del Banco de México. 


\title{
LA INVERSIÓN FIJA EN EL SECTOR MANUFACTURERO MEXICANO 1985-94: EL ROL DE LOS FACTORES FINANCIEROS Y EL IMPACTO DE LA LIBERALIZACIÓN FINANCIERA
}

\author{
Gaston Gelos \\ Alejandro Werner \\ Octubre de 1998
}

\section{RESUMEN}

En este trabajo se estudian los efectos de los factores financieros, como el flujo de caja y el valor del colateral de las empresas, sobre la inversión fija en el sector manufacturero mexicano. Para este fin se utiliza la Encuesta Industrial Anual, recopilada por el INEGI. En un primer análisis, se examina el efecto de diversas variables macroeconómicas sobre la inversión, donde se obtiene el resultado de que la relación entre la tasa de interés real y la inversión cambia de positiva a negativa después del inicio de la liberalización financiera. En la parte principal del estudio, se analiza la importancia de las restricciones de financiamiento, investigando el efecto del flujo de caja y del valor de los bienes raíces (como una medida de colateral) sobre la formación de capital fijo. Estimaciones no paramétricas indican una clara relación entre el flujo de caja y la inversión. Los resultados de un método estándar, en el cual se incluye el flujo de caja en regresiones de inversión convencionales, indican que las restricciones de liquidez influyeron en forma importante sobre la inversión, especialmente en el caso de las empresas pequeñas. Esta clase de empresas, en particular las exportadoras, parecen haber sido las más favorecidas por la liberalización financiera. Mediante un enfoque novedoso, se consideró el alto número de observaciones con cero inversión y la posibilidad de que existan costos fijos en el proceso de inversión, por lo que se estimó un modelo Tobit con efectos fijos incluyendo, además del flujo de caja, el valor de los bienes raíces propiedad de la empresa, como una medida de las garantías con las que cuenta la misma. Se concluye que el valor de los bienes raíces tiene una influencia muy significativa sobre la inversión y que la importancia de este tipo de garantías aumentó después de la liberalización financiera. Debido a esto concluimos que la liberalización se tradujo no tanto en una reducción gradual de la sobretasa del costo de fondos externos sobre el costo de fondos internos, sino más bien en una mayor liquidez en la economía, que recién entonces permitió a muchas empresas el acceso al crédito. 


\section{Contenido}

I. INTRODUCCIÓN

Página

II. EL ENTORNO MACROECONÓMICO, EL SISTEMA FINANCIERO

Y LA INVERSIÓN EN MÉXICO DURANTE EL PERÍODO 1985-94 7

II.1 El entorno macroeconómico: 1985-94 7

II.2 Aspectos del sistema financiero mexicano $\quad 8$

III. MARCO TEÓRICO Y LITERATURA EMPÍRICA 10

III.1 Marco teórico general $\quad 10$

$\begin{array}{ll}\text { III.2 Literatura empírica } & 12\end{array}$

IV. DATOS Y RESULTADOS EMPÍRICOS 14

IV.1 Principales características de los datos utilizados $\quad 14$

IV.2 Resultados empíricos 16

IV.2. 1 La influencia de los efectos agregados $\quad 17$

IV. 2. 2 Los efectos de liquidez antes y después de la liberalización 18

IV.3 El rol de las garantías y la decisión cualitativa de inversión 27

V. CONCLUSIONES

VI. APÉNDICE: Construcción de las variables utilizadas 33

VII REFERENCIAS 


\section{Introducción}

Después de la crisis de 1982, las inversiones en capital fijo del sector privado mexicano disminuyeron considerablemente, manteniéndose en estos bajos niveles hasta el fin de la década, cuando se observa una recuperación importante. Algunos autores han estudiado los determinantes de la inversión privada, concentrándose principalmente en el rol de los términos de intercambio y del "debt overhang" asociado con la crisis de la deuda (véase Warner (1994)).

Nuestra intención en este estudio es analizar los determinantes de la inversión privada durante el período 1985-94, enfocándonos en varios aspectos que presumiblemente tuvieron efectos importantes sobre el comportamiento de ésta: las imperfecciones de los mercados de capitales, la represión financiera y la consiguiente liberalización que se inició a fines del año 1988. ¿ Hasta qué punto las restricciones de liquidez fueron decisivas en la caída de las inversiones ocurridas durante la década de los ochenta? ¿Cómo se vieron afectados los diferentes tipos de empresas antes y después de la liberalización financiera? ¿Las empresas pequeñas y medianas, supuestamente favorecidas por programas de crédito especiales de la banca de fomento, se vieron beneficiadas con la liberalización financiera? En este trabajo se tratan de contestar estas interrogantes.

El énfasis en el rol que juegan los mercados crediticios en países en vías de desarrollo para explicar los niveles de inversión tiene una tradición importante. Los estudios de McKinnon (1973) y Shaw (1973), destacan la importancia del sector financiero en el desarrollo económico. El argumento fundamental que se sostiene en estos trabajos es que el mantenimiento de tasas de interés artificialmente bajas agudiza los problemas de racionamiento de crédito, limitando el proceso de intermediación financiera y el desarrollo económico. En este entorno, la mayoría de las empresas tendrá que financiar sus inversiones a través de fuentes internas.

Por otra parte, en los años recientes ha resurgido el interés en los efectos de las restricciones de liquidez en la determinación de las inversiones en países desarrollados. Esto se debe a que la comprensión del papel de las imperfecciones en los mercados financieros es sumamente relevante para el mecanismo de transmisión monetario. Si la política monetaria puede influir en la sobretasa que enfrentan las empresas entre el costo de fondos externos y el costo de oportunidad de la liquidez interna, esto puede generar un "acelerador financiero". Además, si las empresas dependen del crédito bancario, bajo ciertas circunstancias, el volumen del crédito puede afectar significativamente la actividad económica ${ }^{2}$ por encima de su efecto sobre la tasa de interés. Varios estudios han confirmado mediante el uso de datos desagregados la existencia de importantes efectos-liquidez en la determinación de la

\footnotetext{
${ }^{2}$ Véase Bernanke y Blinder (1988).
} 
inversión a nivel de empresas (para un resúmen de la literatura al respecto, véase Hubbard (1998)).

Actualmente existen pocos trabajos que estudian, con datos a nivel de empresa, el papel de las restricciones de liquidez sobre las inversiones en países menos desarrollados ${ }^{3}$. El caso de México es particularmente interesante debido a los cambios estructurales observados en los últimos diez años. En este estudio se examina la Encuesta Industrial Anual que es una base de datos muy amplia a nivel de planta recolectada por el INEGI entre 1984 y 1994. Esta encuesta abarca alrededor del $80 \%$ del valor agregado en el sector manufacturero. Por otra parte, el trabajo realizado por Babatz y Conesa (1997) también estudia la liberalización financiera en México, pero difiere en el tipo de datos utilizados (71 compañías cotizadas en bolsa) y en el período estudiado (1988-1994)4. Según la teoría, las restricciones de liquidez deberían ser más importantes para empresas pequeñas; por ello, además de la mayor representatividad, es preferible analizar una base de datos que no solamente incluya a compañías cotizadas en bolsa.

Este documento se estructura de la siguiente manera. En la segunda sección se describen a grandes rasgos, las principales características del sistema financiero mexicano y la evolución de la economía durante el período cubierto por el estudio. En la tercera sección se discuten algunas de las predicciones de la literatura acerca de la influencia de las restricciones de liquidez sobre la inversión y los resultados empíricos de otros estudios. La cuarta sección está dedicada a la presentación de las características más relevantes de los datos utilizados, y a la discusión de los resultados empíricos. Además de usar la metodología estándar, se discutirán estimaciones no lineales y los resultados obtenidos mediante un método innovador que nos permite diferenciar la decisión empresarial cualitativa de invertir o no invertir, de la decisión cuantitativa acerca del volumen de la inversión. En la quinta sección se presentan las conclusiones. Detalles acerca de los datos utilizados se encuentran en el apéndice.

Los resultados demuestran que para las empresas chicas y medianas, efectivamente la liquidez interna fue un factor importante en la determinación de los niveles de inversión durante todo el período considerado. Por otra parte, la liberalización financiera contribuyó a reducir las restricciones de liquidez de estas compañías. Un resultado interesante con respecto al papel de las garantías y del

\footnotetext{
${ }^{3}$ Entre las excepciones se encuentran Tybout (1983), Nabi (1989), Athey y Laumas (1994), Harris, Schiantarelli y Siregar (1994) y Jaramillo y Schiantarelli (1997) así como Babatz y Conesa (1997). Los estudios de Harris et al. (1994) y Jaramillo et al. (1997), que analizan los efectos de la liberalización financiera en Indonesia y Ecuador, respectivamente, son los más similares al presente estudio tanto en el enfoque como en el tipo datos utilizados.

${ }^{4}$ Como se discutirá más adelante, la liberalización financiera se inicia a fines de 1989, lo cual hace difícil la interpretación de los resultados de Babatz y Conesa (1997).
} 
colateral (terrenos y edificios) en la decisión de inversión, es que la probabilidad de realizar una inversión crece rápidamente, ceteris paribus, con la proporción de bienes raíces en el capital fijo total de la empresa. También, se detectó cierta evidencia de que las empresas con un acervo importante de este tipo de garantías se ven sujetas a menores restricciones de liquidez. 


\section{EL ENTORNO MACROECONÓMICO, EL SISTEMA FINANCIERO Y LA INVERSIÓN EN MÉXICO DURANTE EL PERÍODO 1985-94}

\section{II.1 El entorno macroeconómico: 1985-94}

Después de la grave crisis de 1982, México pasó de ser un importador neto de capitales a ser un exportador neto. Durante el período 1981-1985, las transferencias netas de recursos del exterior cayeron del $7.4 \%$ del PIB a $-5.8 \%{ }^{5}$. Esta circunstancia influyó decisivamente en el programa de reformas económicas implementado por el gobierno a partir de 1982. El objetivo primordial de dichas reformas fue el restablecimiento del equilibrio externo y el control de los efectos inflacionarios de este ajuste, para lo cual se llevó a cabo una reducción sustancial del déficit fiscal. Mientras que el déficit operacional bajó rápidamente, la inflación se redujo de una manera paulatina. Con la intención de contener el crecimiento de los precios, se controló la tasa de depreciación del tipo de cambio, generando como resultado una apreciación real de la moneda. Los terremotos de 1985 dañaron seriamente a la economía, y la situación se empeoró con el choque adverso en los términos de intercambio provocado por la caída del precio internacional del petróleo en 1986. En este año el PIB cayó en un 3.5 \% y la inversión total bajó del $21.2 \%$ al $18.2 \%$ del producto. Como respuesta a estos factores, se adoptó una política de tipo de cambio real más agresiva, que consistió en acelerar la tasa del desliz cambiario. Esto provocó un aumento en la tasa de inflación y culminó en una severa crisis financiera en 1987. El mejoramiento de la competividad favoreció al sector exportador manufacturero, mientras que la contracción de la demanda interna ejerció un efecto dañino sobre el sector no-exportador.

A finales de 1987, al enfrentarse con una inflación creciente (159.2\% en ese año), el gobierno firmó un acuerdo con los tres grupos económicos más importantes del país: empresarios, obreros y campesinos. El llamado Pacto de Solidaridad Económica tenía como propósito corregir la inercia salarial, acordar precios en ciertos sectores, continuar con el saneamiento de las finanzas públicas, mantener una política monetaria restrictiva y acelerar el proceso de apertura comercial. En el ámbito cambiario, se adoptó durante el primer año del programa un tipo de cambio fijo, seguido por un desliz predeterminado del tipo de cambio. Además, se inició un importante programa de privatización y se comenzó con el cierre de compañías deficitarias controladas por el Estado. Como resultado, la inflación disminuyó a 19.7\% en 1989; y el superávit primario del sector público alcanzó el $8 \%$ del PIB. En lo que respecta al desempeño de la inversión total también se pueden apreciar efectos positivos, ya que ésta recuperó el nivel observado en 1985. Sin embargo, las altas tasas de interés, debidas en parte a la incertidumbre acerca de la inflación futura, tuvieron un efecto negativo sobre el crecimiento de las inversiones.

\footnotetext{
${ }^{5}$ Véase Aspe (1993), p. 22.
} 
Durante el periodo 1988-1990 el producto recuperó su dinamismo y la tasa de crecimiento del PIB pasó de $1.3 \%$ en 1988 al 3.1\% en 1989 y a $4.4 \%$ en 1990 . Al mismo tiempo, se renegoció la deuda externa bajo el plan Brady, lo cual llevó a una importante disminución del servicio de la deuda como porcentaje del PIB, a una repatriación de capitales, y a un descenso considerable de las tasas de interés internas.

No obstante los éxitos de las reformas, a principios de los noventa se comenzó a notar una desaceleración de la economía, una apreciación del tipo de cambio real y un déficit creciente en la cuenta corriente. Para 1993 el crecimiento del PIB fue de sólo 0.6 \%. En el año de 1994 el crecimiento del producto recupera su tendencia, pero el año culmina con la crisis cambiaria del 19 de diciembre.

Los factores discutidos se ven reflejados en la evolución de la inversión fija que se muestra en la gráfica 1 .

\section{Gráfica 1 \\ Índice de Volumen de la Inversión Fija Bruta}

$(1980=100)$

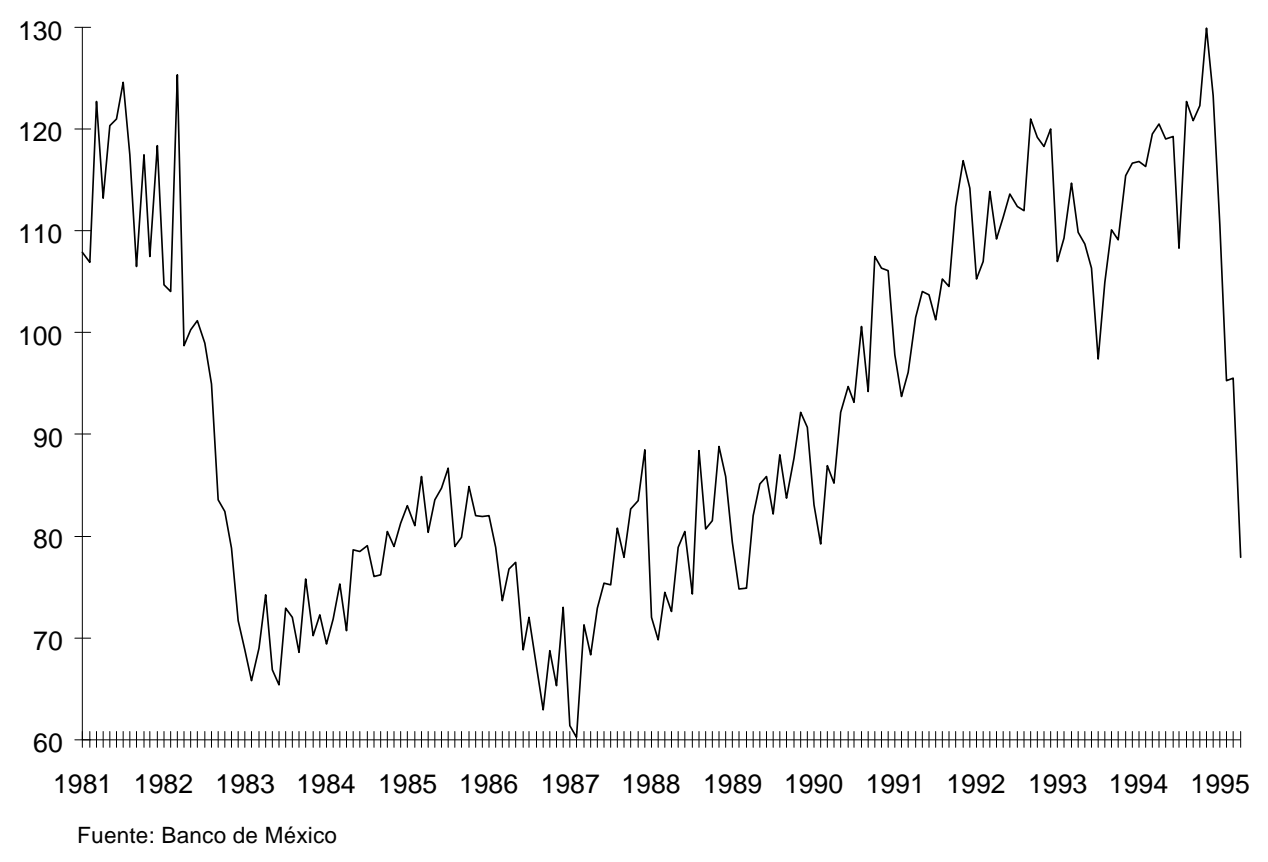




\section{II.2 Aspectos del Sistema Financiero Mexicano}

Hasta 1988, el sistema bancario que había sido nacionalizado en 1982 durante el gobierno de José López Portillo, estaba estrictamente regulado. La intermediación financiera se vió limitada por altos encajes legales, la obligación de los bancos de comprar deuda del estado y la imposición de controles a las tasas de interés pagadas sobre los depósitos. Con altas tasas de inflación, las tasas de interés reales sobre depósitos bancarios fueron negativas, resultando en una baja captación de ahorros privados por el sistema bancario ${ }^{6}$. Mientras que el flujo de crédito de la banca al sector privado se redujo sensiblemente en 1985, los requerimientos de financiamiento del sector público se incrementaron. En 1986, más del sesenta por ciento del crédito neto bancario estaba destinado al gobierno. El capital fluyó en gran parte al extranjero o se invirtió improductivamente, como por ejemplo en metales preciosos o bienes inmobiliarios ${ }^{7}$. Las empresas, por su parte, enfrentaron parcialmente el problema de liquidez con el desarrollo de un mercado de crédito interempresarial no regulado ${ }^{8}$.

En 1988, se inició un marcado proceso de liberalización financiera, que incluyó la liberalización de las tasas de interés, la eliminación de cuotas crediticias y préstamos forzados y la abolición de reservas obligatorias. Este proceso se había completado en su mayor parte en 1990, aunque es en 1992 cuando se cierra el mismo con la privatización de la banca. Junto con la reducción del déficit fiscal, a la que se hizo mención anteriormente, el gobierno comenzó a financiarse principalmente a través de la emisión de CETES a tasas de mercado. Estas reformas se tradujeron en un importante "crowding in" del sector privado. El ahorro captado por el sector bancario se incrementó fuertemente, y el crédito al sector privado creció de manera significativa. El renovado flujo de capital extranjero fue un factor adicional que ayudó a incrementar la disponibilidad de crédito para el sector privado. Después de alcanzar su nivel más bajo en 1987, el flujo de crédito bancario al sector privado aumentó $30 \%$ en términos reales en el último semestre de 1988, mientras que el flujo correspondiente al sector público disminuyó considerablemente.

Estas tendencias también se reflejaron en el crédito al sector manufacturero. En la gráfica 2 se puede apreciar el crédito como porcentaje del PIB sectorial otorgado a este sector por la banca comercial y por la de fomento. Mientras que el crédito de la banca de desarrollo va disminuyendo a partir de 1987, el crédito de la banca comercial se incrementa sensiblemente a partir de 1989. En la próxima sección se discutirá el marco formal en el cual se analizarán más a fondo estos fenómenos para entender en qué forma y medida el aumento del crédito bancario dió

\footnotetext{
${ }^{6}$ Véase Babatz y Conesa (1997) y Gruben y McComb (1997).

${ }^{7}$ Véase Mansell Carstens (1995), p. 17.

${ }^{8}$ Véase Skiles (1991).
} 
lugar a una disminución de las restricciones financieras que enfrentaban las empresas individuales.

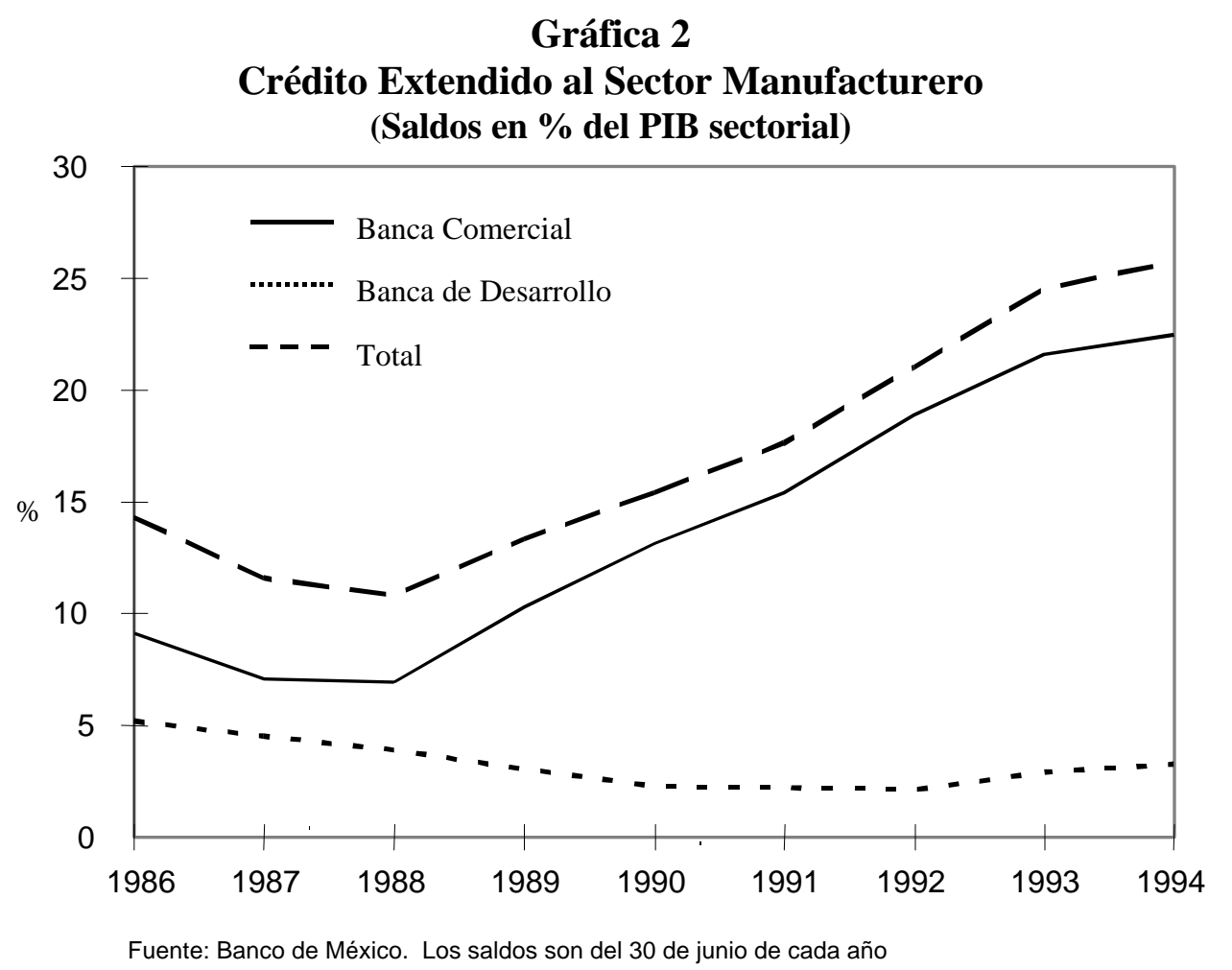

\section{Marco teórico y literatura empírica}

\section{III.1 Marco teórico general}

La importancia del sistema financiero para el desarrollo económico ha sido subrayada por McKinnon (1993) y Shaw (1973) ${ }^{9}$. Recientemente, el impacto del desarrollo de los mercados de capitales sobre el crecimiento de la productividad ha sido incorporado a las nuevas teorías de crecimiento económico y su importancia ha sido confirmada en varios trabajos empíricos (véase por ejemplo la discusión en Levine (1997)).

En el marco original de McKinnon, la fragmentación de los mercados financieros conduce a una asignación subóptima del capital, lo que impacta de manera negativa al crecimiento económico. Estos problemas son exacerbados por la llamada "represión financiera", un fenómeno muy común en países en vías de desarrollo y descrito por McKinnon (1993) y Shaw (1973). La represión financiera se caracteriza por topes a las tasas de interés en combinación con altas tasas de inflación, elevados encajes legales y políticas de crédito selectivas. Las medidas

${ }^{9}$ Para una discusión en el contexto de México, véase Ernst Dolgow (1991) y Mansell Carstens (1995). 
mencionadas desalientan el ahorro, la toma de riesgo por parte de las instituciones financieras y conducen a que no se financien inversiones con altas tasas de rendimiento.

Por otra parte, en años recientes se ha desarrollado una importante literatura que analiza las consecuencias de las asimetrías de información y otro tipo de imperfecciones, como los costos de quiebra y la existencia de responsabilidad limitada, sobre el crédito y la inversión. Estos avances teóricos han aumentado el interés sobre las implicaciones de las imperfecciones de los mercados financieros en países desarrollados.

En un mundo sin asimetrías de información, con un sistema legal perfecto, sin costos de quiebra y sin impuestos, la decisión empresarial acerca de la inversión se puede separar por completo de la decisión concerniente a su financiamiento debido al teorema de Modigliani y Miller (1958). Por otra parte, en un entorno con un sistema legal imperfecto y con asimetrías de información entre empresarios y acreedores, el costo para las empresas del financiamiento externo siempre será más alto que el costo de los fondos propios (véase Bernanke, Gertler y Gilchrist (1996)). Stiglitz y Weiss (1981) han desarrollado un modelo que describe el caso extremo de racionamiento de crédito. En ese modelo, la tasa de interés no se mueve para equilibrar la oferta y la demanda de crédito. Más bien, debido a diferencias entre la información de deudores y empresarios, los bancos limitan la cantidad de crédito sin aumentar la tasa de interés para evitar que los prestamistas se vean incentivados a invertir en proyectos demasiado riesgosos. En este caso, si las empresas no pueden recurrir a otro tipo de financiamiento, se puede decir que la sobretasa del costo de fondos externos (a la empresa) sobre el costo de fondos internos es infinita.

Generalmente, las condiciones a las cuales los acreedores estarán dispuestos a otorgar crédito, dependerán del valor neto de la empresa, o de las garantías que ésta pueda ofrecer. En otras palabras, la sobretasa del costo de fondos externos sobre el costo de oportunidad del propio flujo de caja disminuirá con una mejor situación financiera de la empresa. (Véase por ejemplo Townsend (1979), Bernanke y Gertler (1989) y Bernanke y Gertler (1997)). Nótese que la noción de una sobretasa de los costos externos sobre los costos internos de financiamiento no se limita a los contratos de deuda, sino que argumentos similares también se pueden hacer con respecto a la emisión de papeles comerciales o de acciones ${ }^{10}$.

Esta observación tiene una consecuencia importante: acontecimientos o medidas económicas que afecten al valor neto de las empresas pueden tener un impacto significativo sobre la inversión. Por ejemplo, una política monetaria contraccionista (como por ejemplo un incremento de la tasa de interés) puede reducir

\footnotetext{
${ }^{10}$ Véase Myers y Majluf (1984).
} 
el valor de las empresas, aumentando el costo de financiamiento, y disminuyendo la inversión por un canal de transmisión adicional al tradicionalmente descrito en los libros de texto. Cabe señalar que la misma medida de política monetaria puede diferir en su efecto, dependiendo de la situación financiera de las empresas. Bernanke y Gertler (1989), Kiyotaki y Moore (1997), y, más recientemente Bernanke y Gertler (1997), desarrollan modelos que demuestran cómo, a partir del supuesto de información asimétrica, se puede llegar a un acelerador financiero que amplifica los ciclos económicos ${ }^{11}$.

Para países en desarrollo, muchas veces se menciona que el racionamiento de crédito es más importante que la noción de que el costo de crédito disminuya con el valor neto de la empresa. El argumento se debe a que en el mejor de los casos las compañías tienen acceso a un volumen de crédito determinado, a una tasa de interés fija $^{12}$. En el caso de México, lo más probable es que una combinación de varios efectos explique las restricciones de liquidez. Por un lado, es cierto que muchas empresas se vieron verdaderamente incapacitadas para acceder a algún tipo de fondos, especialmente a mediados de los ochenta. Por otra parte, la banca de fomento otorgó créditos en condiciones favorables, contribuyendo en algunos casos, al financiamiento de inversiones. Debido a esto creemos que dependerá del tamaño y de otras características de la compañía, si los costos de fondos adicionales se pueden caracterizar como infinitos o meramente crecientes con el endeudamiento de la empresa.

\section{III.2. Literatura empírica}

La literatura empírica se ha concentrado principalmente en encontrar evidencia acerca de los efectos de la liquidez sobre la inversión a nivel de empresas. La idea es que cuando existen restricciones de liquidez, se debería observar una importante relación entre los fondos internos de las empresas y el nivel de sus inversiones. Si las empresas no enfrentan restricciones de liquidez, la adición de variables financieras a un modelo econométrico de inversión correctamente especificado no tendría que mejorar la predicción del nivel de la inversión. Por otra parte, si el costo de fondos externos para las empresas es muy alto, el flujo de caja de una empresa ayudará a predecir sus inversiones, ya que éstas serían en su mayor parte autofinanciadas. Aunque desde el estudio de Meyer y Kuh (1957) se documentó la conexión entre el flujo de caja empresarial y la inversión, éstos resultados eran difíciles de interpretar. Esto se debe a que un flujo de caja alto

\footnotetext{
${ }^{11}$ Nótese que en el caso que, a raíz de los problemas de información mencionados, las empresas solamente puedan acceder al crédito bancario como único financiamiento, la política monetaria puede transmitirse por un canal de crédito, siempre y cuando los bancos no puedan fácilmente sustituir créditos por otros activos en su cartera (véase Bernanke y Blinder (1988)).

${ }^{12}$ Véase Rama (1993).
} 
puede reflejar un aumento en la rentabilidad futura, lo que también induciría un aumento en la inversión en los modelos con mercados de capitales perfectos.

Fazzari, Hubbard y Peterson (1988) emplean una técnica más sofisticada para discernir los efectos liquidez. Además de incluir la q de Tobin como variable que captura la rentabilidad futura esperada en sus regresiones de inversión, dividen las empresas en diferentes categorías, que a priori tendrían que diferir en el grado en el cual enfrentan restricciones de liquidez. Específicamente, clasifican como restringidas aquellas empresas, que, a pesar de haber realizado ganancias, han distribuido pocos dividendos. Los resultados empíricos demuestran que, para estas compañías, el coeficiente del flujo de caja en las regresiones de inversión es más alto que para las empresas que fueron clasificadas ex-ante como menos restringidas ${ }^{13}$.

A partir de este trabajo, esta estrategia de identificación ha sido utilizada por muchos autores ${ }^{14}$. Gilchrist y Himmelberg (1996) realizan otro intento econométrico para tomar en cuenta la información sobre de la rentabilidad futura contenida en el flujo de caja: estiman un vector autorregresivo (VAR), incluyendo al flujo de caja como una de las variables, permitiendo así una proyección de la rentabilidad futura sobre las variables contemporáneas.

En algunos casos, el análisis de diferencias entre grupos de empresas se ha combinado con el estudio de cambios a través del tiempo. Gertler y Gilchrist (1994) examinan el comportamiento de empresas pequeñas y grandes después de perturbaciones monetarias contractivas. Harris et al. (1994), Babatz y Conesa (1997) y Jaramillo et al. estudian los efectos de la liberalización financiera en Indonesia, México y Ecuador, respectivamente. Finalmente, el trabajo de Schiantarelli (1996) resume la literatura en forma exhaustiva.

También existe una literatura al respecto que utiliza datos agregados, aunque la interpretación de estos resultados muchas veces es problemática. Dailami y Giugale (1991), por ejemplo, formulan el problema de optimización con restricciones de liquidez para la empresa individual, pero utilizan datos agregados de Brasil, Colombia, India, Corea y Turquía para analizar la relación entre el crédito y la inversión. Para el caso de México, Gunther y Moore (1993), Copelman y Werner (1997) y Greenham (1997) estiman vectores autorregresivos (VAR's) para discernir los efectos del crédito sobre la actividad macroeconómica. En los dos últimos trabajos se establece la existencia de un canal de crédito para México, lo cual presupone importantes restricciones de financiamiento a nivel de empresa.

\footnotetext{
${ }^{13}$ Kaplan y Zingales (1997) critican la metodología empleada por Fazzari et al. y aceveran que teóricamente la relación entre la magnitud del coeficiente sobre el flujo de caja y el grado de restricción de liquidez es ambigua.

${ }^{14}$ Tybout (1983) en un principio ya había empleado una metodología similar al diferenciar entre empresas pequeñas y grandes.
} 


\section{DATOS Y RESULTADOS EMPIRICOS}

\section{IV.1. Principales Características de los Datos Utilizados}

La muestra analizada es la base de datos de la "Encuesta Industrial Anual" recolectada por el INEGI. Esta muestra cubre 3199 establecimientos, encuestados desde 1984 hasta 1994, e incluye alrededor del $80 \%$ del valor agregado en el sector manufacturero. En la selección de los establecimientos de la muestra se dió preferencia a las empresas mayores; a pesar de esto, la base incluye un gran número de empresas con un personal empleado menor a veinte personas. Los datos proporcionados comprenden una amplia gama de variables; la muestra es balanceada en el sentido de que para cada año el número de plantas es el mismo ${ }^{15}$.

Una desventaja para los fines de este trabajo se debe a que la información recogida es a nivel de plantas, mientras que para nuestro análisis, especialmente con respecto al flujo de caja, la unidad más relevante es la empresa. Esta dificultad se superó parcialmente utilizando para la construcción del flujo de caja las utilidades repartidas que es una variable que contiene información acerca de las utilidades a nivel de empresa. Los detalles acerca de la construcción de las variables se encuentran en el apéndice. Allí también se describe como de la base de datos inicial, que comprende a 3199 plantas, se eliminaron establecimientos con datos extremos, incompletos o inconsistentes. La muestra final analizada comprende 834 establecimientos.

Las plantas se dividieron en tres categorías con respecto a su tamaño. Se denominan "pequeñas" aquéllas que emplean menos de cien personas, "medianas" a las que emplean entre 100 y 500 personas, y "grandes" a las plantas con más de 500 personas. Como el período estudiado es de diez años, la clasificación de una planta puede cambiar, dependiendo de su crecimiento. Hasta 1990 se cuenta con información acerca de los propietarios de las plantas, por lo tanto se puede diferenciar entre privadas, con participación estatal y públicas, así como entre aquéllas con participación extranjera ${ }^{16} \mathrm{o}$ sin ella.

En el cuadro 1 se presentan las principales características de los establecimientos analizados.

\footnotetext{
${ }^{15}$ Según infomación de INEGI, los establecimientos entrantes y salientes de la encuesta original fueron muy pocos.

${ }^{16}$ Asumimos que, después de 1990, los establecimientos mantuvieron el status con respecto a su estructura de propiedad que tenían en 1990. Esto puede ser erróneo debido a las privatizaciones que se llevaron a cabo después de ese año.
} 
Es importante destacar que, debido al método utilizado para su construcción, el flujo de caja construido reflejará (con las restricciones discutidas más adelante) el verdadero valor sólo en aquellos casos en los cuales las utilidades de la empresa hayan sido positivas. Por lo tanto, los números indicados en la tabla tienden a sobrestimar el valor de esta variable (aunque las observaciones con cero utilidades repartidas son muy pocas).

\section{Cuadro 1}

\begin{tabular}{|c|c|c|c|c|c|}
\hline & $\begin{array}{l}\text { Número } \\
\text { de } \\
\text { observ. }\end{array}$ & $\begin{array}{l}\text { Personal } \\
\text { total }\end{array}$ & $\begin{array}{l}\text { Acervo de } \\
{\text { Capital } K_{t}}^{2}\end{array}$ & $\begin{array}{l}\text { Inversión } \\
\mathbf{I}_{\mathbf{t}} / \mathbf{K}_{\mathrm{t}-1}\end{array}$ & $\begin{array}{l}\text { Flujo de caja } \\
\mathrm{CF}_{\mathfrak{t} / \mathbf{K}_{\mathrm{t}-1}{ }^{*}}\end{array}$ \\
\hline $\begin{array}{l}\text { Pequeñas } \\
\text { privadas }\end{array}$ & 2781 & 54 & 6800 & 0.07 & 0.16 \\
\hline & & (24) & (14950) & $(0.14)$ & $(0.25)$ \\
\hline con part. estatal & 62 & $\begin{array}{l}52 \\
(22)\end{array}$ & $\begin{array}{l}76300 \\
(166100)\end{array}$ & $\begin{array}{l}0.08 \\
(0.14)\end{array}$ & $\begin{array}{l}0.18 \\
(0.29)\end{array}$ \\
\hline $\begin{array}{l}\text { Medianas } \\
\text { privadas }\end{array}$ & 4556 & 233 & 32500 & 0.07 & 0.18 \\
\hline & & (108) & $(54100)$ & $(0.11)$ & $(0.27)$ \\
\hline con part. estatal & 149 & $\begin{array}{l}261 \\
(98)\end{array}$ & $\begin{array}{l}671900 \\
(80800)\end{array}$ & $\begin{array}{l}0.05 \\
(0.10)\end{array}$ & $\begin{array}{l}0.08 \\
(0.14)\end{array}$ \\
\hline $\begin{array}{l}\text { Grandes } \\
\text { privadas }\end{array}$ & 1542 & $\begin{array}{l}1046 \\
(1136)\end{array}$ & $\begin{array}{l}159250 \\
(274600)\end{array}$ & $\begin{array}{l}0.08 \\
(0.12)\end{array}$ & $\begin{array}{l}0.19 \\
(0.27)\end{array}$ \\
\hline con part. estatal & 84 & $\begin{array}{l}1583 \\
(1778)\end{array}$ & \begin{tabular}{|l}
273700 \\
$(391300)$
\end{tabular} & $\begin{array}{l}0.04 \\
(0.06)\end{array}$ & \begin{tabular}{|l|}
0.09 \\
$0.17)$
\end{tabular} \\
\hline
\end{tabular}

Nota: El número de observaciones indica el número de años-planta en esta clasificación. Los acervos de capital están indicados en miles de pesos del año 1994. Los números de la segunda a la quinta columna denotan el promedio y la desviación estándar (entre paréntesis), de las variables mencionadas. El flujo de caja fue construido con base en las utilidades repartidas reportadas y a la depreciación.

Las empresas con participación estatal, tienden a tener un acervo de capital más alto que las privadas, independientemente de su tamaño. Sin embargo, las empresas estatales de tamaño mediano y grande tienden a invertir menos que las privadas y sus utilidades son menores. Finalmente, se observa que las grandes empresas privadas tienen utilidades y tasas de inversión ligeramente mayores a las observadas en las empresas pequeñas y medianas. 


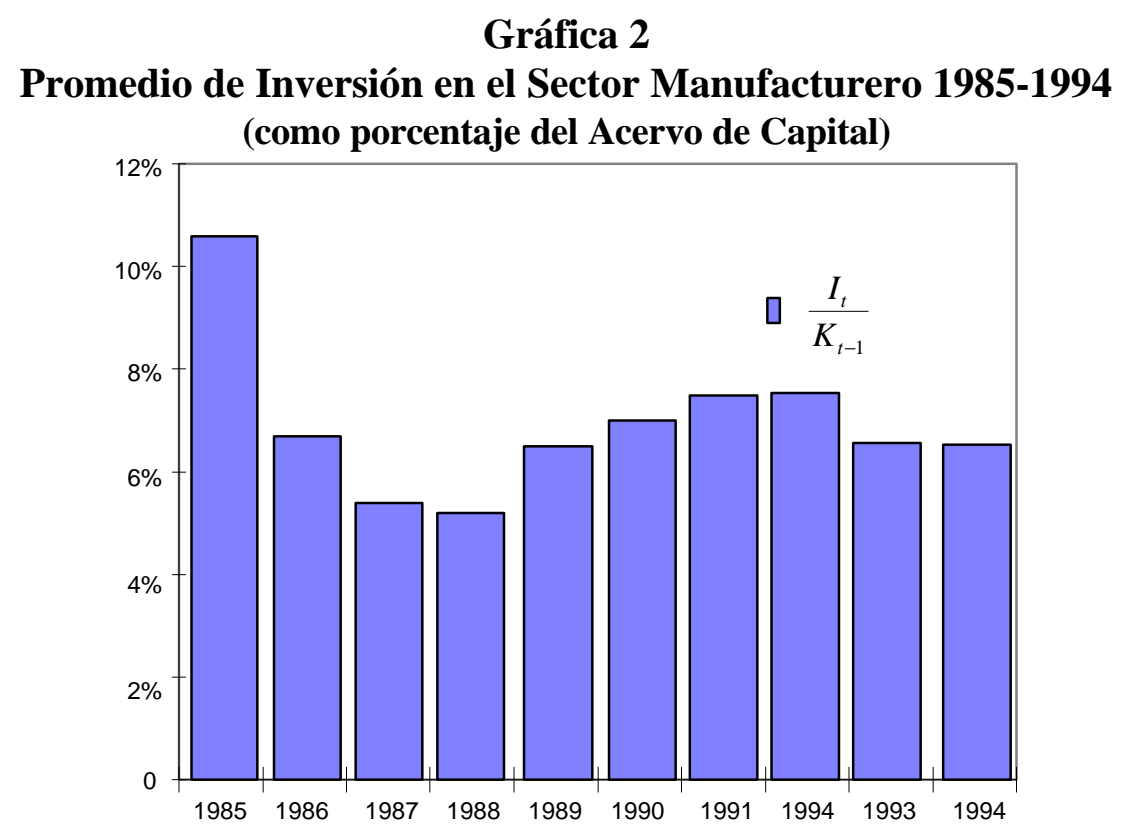

La inversión en la muestra utilizada refleja parcialmente el movimiento de la inversión fija privada total ${ }^{17}$ de cuentas nacionales; la diferencia más marcada se muestra en la fuerte caída que tiene lugar en nuestra muestra entre 1986 y 1988, ésta no se presenta en los datos de cuentas nacionales (en la gráfica 1). A partir del inicio de las reformas de 1989, la inversión se recupera lentamente durante los años siguientes, pero nunca alcanza el nivel inicial. En 1993 y 1994 se registra una ligera desaceleración en la formación de capital, debido a los factores macroeconómicos discutidos en la sección II. Nótese que las tasas de inversión son bajas, y en varios años es dudoso que excedan a la tasa de depreciación.

\section{IV.2. Resultados empíricos}

Las especificaciones econométricas más utilizadas para explicar los niveles de inversión son las de tipo acelerador y las que utilizan la q de $\operatorname{Tobin}^{18}$. En el caso presente, el modelo de acelerador es la especificación indicada debido a la imposibilidad de calcular la q de Tobin, para la cual se necesitaría que todas estas empresas cotizaran en la bolsa. Por esa razón, la especificación básica utilizada en el análisis es la siguiente:

$\frac{I_{i t}}{K_{i, t-1}}=\beta \frac{\Delta y_{i t}}{K_{i t-1}}+$ Variables adicionales

\footnotetext{
${ }^{17}$ La comparación no es inmediata, ya que en el gráfico 1 se utiliza el PIB como variable de escala, mientras que aquí se divide por el acervo de capital rezagado.

${ }^{18}$ Alternativamente, en la literatura se han estimado ecuaciones de Euler (véase Schiantarelli (1996)). El problema con estas estimaciones es que son muy susceptibles a errores de especificación.
} 
Donde $K_{i t}, y_{i t}$ denotan al acervo de capital y la producción de la empresa i en el período t, respectivamente y $\beta$ mide la sensibilidad de la inversión ante cambios en la producción. Las variables adicionales que se incorporarán se discutirán en las secciones siguientes.

\section{IV.2.1 La influencia de los efectos agregados}

En un análisis preliminar, examinamos la respuesta de la inversión en las plantas de nuestra muestra a variaciones en algunas variables agregadas como el crecimiento del PIB, la tasa de interés real, y el tipo de cambio real. Naturalmente, este ejercicio es de interés limitado, ya que no podemos hacer uso de variaciones en el corte transversal en estas variables. No se logró relacionar el efecto del tipo de cambio con la información acerca de la estructura de exportación o importación de la empresa, en el sentido propuesto por Goldberg (1996).

Para obtener un cierto control econométrico sobre la influencia de choques de rentabilidad idiosincrática, también incluimos el cambio en la producción a nivel de planta, $\frac{\Delta y_{i t}}{K_{i t-1}}$, como variable adicional. Además, incluímos efectos fijos individuales para evitar problemas asociados con heterogenidad no observada.

Se esperaría que la tasa de crecimiento del PIB tuviera un efecto positivo sobre la inversión, debido a su carácter de señal en relación al clima económico general. Con respecto al efecto esperado de la tasa de interés real (r), éste es más ambiguo. Por una parte, el marco teórico discutido previamente sugeriría que el costo de fondos varíe de empresa a empresa, dependiendo de sus carácterísticas individuales, con lo cual una variable agregada no sería muy informativa. Por otra parte, se esperaría que durante la época de represión financiera, con tasas reales negativas y racionamiento de crédito, la tasa real no haya sido la variable determinante en la decisión de inversión. Mas bien, durante ese período, las tasas de interés reales mayores estarían más correlacionadas con tasas de inflación menores y una disponibilidad de crédito mayor, sugiriendo una relación positiva entre la inversión y la tasa de interés real antes de 1988. Si la liberalización financiera realmente aumentó el acceso a fondos, a partir de 1989, se debería observar una mayor relación "neoclásica" negativa entre la tasa de interés y la inversión.

El tipo de cambio real (TCR), representa un precio relativo importante que ha variado considerablemente durante el período examinado. Esta variable presumiblemente influyó las inversiones realizadas por varias vías. Por una parte, en México, las devaluaciones siempre han sido contractivas y además, dado que una gran parte de los bienes de inversión son importados, las depreciaciones del TCR 
están asociadas con aumentos en el precio relativo de los bienes de inversión. Por otra parte, los sectores exportadores se ven beneficiados por las depreciaciones de la moneda.

Tabla 2: La influencia de efectos agregados - estimación con efectos fijos

\begin{tabular}{c|ccccc}
\hline Variable & $\Delta y_{i t}$ & Crecimiento PIB & $r$ & $r$ después 88 & TCR \\
& $K_{i t-1}$ & & & & \\
\hline Coeff. & 0.01 & 0.22 & 0.001 & -0.001 & -0.001 \\
(Estad. t) & $(4.17)$ & $(3.05)$ & $(6.57)$ & $(-7.66)$ & $(-1.48)$ \\
\hline
\end{tabular}

No. de observaciones: $8340 \mathrm{R}^{2}=0.02 \mathrm{~F}(6,7500)=24.3$ Un aumento del TCR es equivalente a una depreciación.

Se puede apreciar que, además de choques idiosincráticos a la demanda $\left(\Delta y_{i t} / K_{i t-1}\right)$ de cada empresa, la tasa de crecimiento del PIB está asociada significativamente con la inversión. La tasa de interés promedio tiene un impacto positivio previo a 1988, mientras que ese efecto desaparece después de 1988, como habíamos predecido. La correlación con el tipo de cambio real no es definida, lo cual tampoco es sorprendente como se discutió previamente.

\section{2. 2. Los efectos de liquidez antes y después de la liberalización financiera}

Para obtener una noción preliminar de la relación entre la inversión y el flujo de caja empresarial, utilizamos un método no paramétrico, sin imponer restricciones funcionales. Dado que nuestro interés no se enfoca en los efectos agregados, en un primer paso, eliminamos los efectos debidos a variaciones en las condiciones macroeconómicas, corriendo una regresión de la inversión sobre dummies anuales. En un segundo paso, relacionamos los residuos de esta estimación con el flujo de caja empresarial computando una relación no lineal con un estimador "kernel", diferenciando entre empresas pequeñas por un lado, y compañías medianas y grandes por el otro. Las inversiones deberían estar más influenciadas por el flujo de caja en el caso de los establecimientos pequeños, los cuales probablemente han tenido más dificultades en acceder a fondos externos. El argumento más común que avala este supuesto es que existen economías de escala en el procesamiento y la divulgación de información que ayudan a sobrellevar los problemas asociados con las asimetrías de información. Las empresas pequeñas también son, en promedio, aquéllas con mayores riesgos idiosincráticos y con pocas garantías ${ }^{19}$.

El tipo de regresión utilizado practicamente no impone restricciones sobre la función estimada, y es útil para detectar no linearidades. Para cada flujo de capital, la estimación calcula un promedio de las tasas de inversión observadas en su vecindad, con pesos dados por el kernel ${ }^{20}$. Aquí utilizamos el estimador Nadarya-

\footnotetext{
${ }^{19}$ Véase Gertler y Gilchrist (1994).

${ }^{20}$ Véase Hardle (1990) para más detalles.
} 
Watson con un kernel Epachenikov y una amplitud de banda de 0.05. Los resultados se pueden apreciar en los gráficos 3 y $4^{21}$.

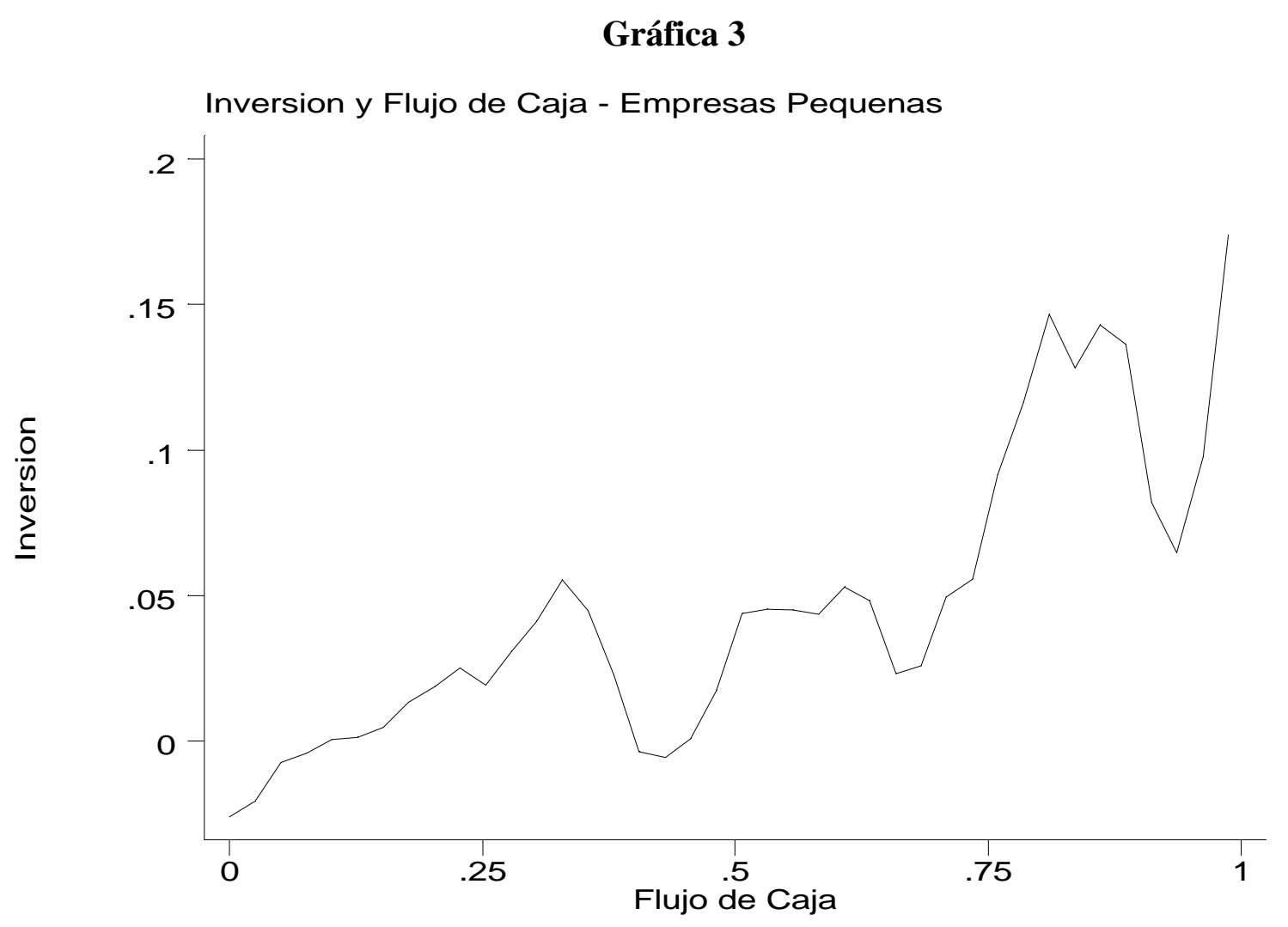

\footnotetext{
${ }^{21}$ La gran mayoría de las observaciones del flujo de caja no exceden valores mayores al cien por ciento del valor del acervo de capital. Suprimimos valores de flujo de caja mayores para evitar obtener gráficas distorcionadas debido a un número de observaciones muy pequeño.
} 


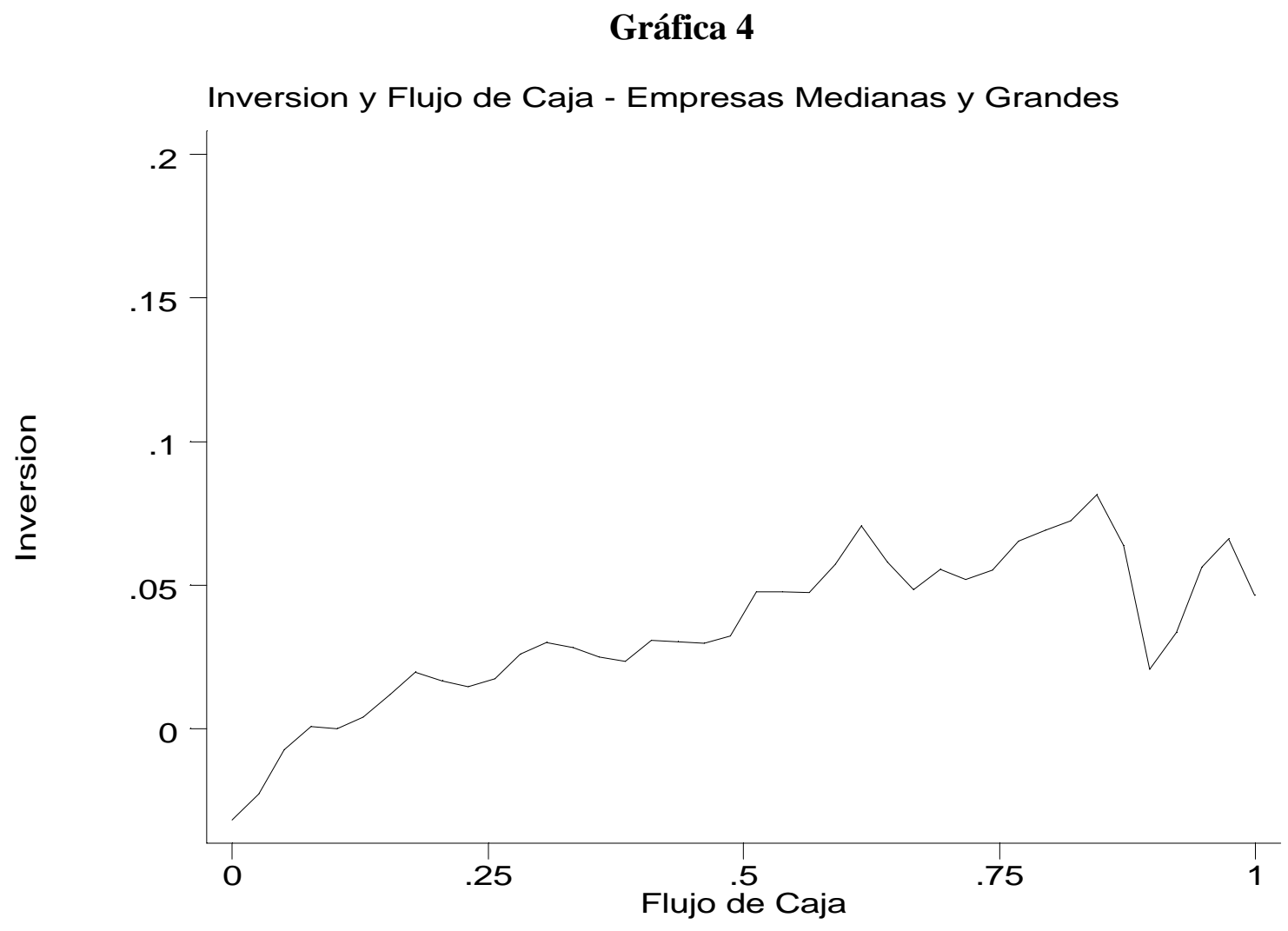

En los dos casos, se observa una relación pronunciada entre ambas variables. Sin embargo, como habíamos esperado, incrementos en el flujo de caja están asociados con aumentos en la tasa de inversión mucho mayores en el caso de las empresas pequeñas. Los gráficos también demuestran que, sobre todo en el caso de la empresas medianas y grandes, es posible aproximar la función satisfactoriamente mediante una relación lineal. Por lo tanto, en los siguiente continuaremos basando nuestras estimaciones en la forma funcional convencional presentada en (1).

La especificación básica será:

$\frac{I_{i t}}{K_{i t-1}}=\beta \frac{\Delta y_{i t}}{K_{i t-1}}+\phi \frac{C F_{i t}}{K_{i t-1}}+\lambda_{t}+v_{i}+\varepsilon_{i t}$

Donde $\varepsilon_{i t}$ denota un error estocástico. Aquí $v_{i}$ denotan efectos específicos para cada empresa y $\lambda_{r}$ dummies anuales, finalmente, el parámetro $\phi$ mide la sensibilidad de la inversión al flujo de caja. De esta forma se controla por cambios en el entorno macroeconómico, y otros factores que no se incluyen explícitamente en la regresión. Para estudiar el efecto de las restricciones de liquidez, se incluye el flujo de caja $\left(\mathrm{CF}_{\mathrm{it}}\right)$ como una variable explicativa adicional. 
Si los efectos específicos a la empresa, $v_{i}$, no están correlacionados con las variables explicativas, esta ecuación se podría estimar con un modelo de "efectos aleatorios". Por otro lado, si los efectos están correlacionados con las variables explicativas, se optaría por una estimación con efectos fijos. Las pruebas de Hausman indican la presencia de correlación, y por ello solamente se presentan los resultados de la estimación con efectos fijos ${ }^{22}$.

En las estimaciones se deja variar el coeficiente del flujo de caja para empresas de diferentes tamaños. Para esto se crearon variables de interacción entre las dummies para las tres clases de empresas de acuerdo a su tamaño y el flujo de caja.

Además se distingue entre varias otras clases de plantas: las privadas y las empresas con participación estatal, aquéllas con participación extranjera y las puramente nacionales y finalmente, las exportadoras y las no exportadoras. Se espera que los coeficientes del flujo de caja para las empresas privadas sean mayores que para las empresas con participación estatal, ya que estas últimas probablemente se beneficiaron más del otorgamiento de créditos selectivos y cuentan con garantías implícitas del gobierno. Además, en el caso de las empresas con participación estatal, se espera un aumento de la correlación entre la liquidez interna y la inversión, después de la liberalización financiera, mientras que se espera observar lo contrario en el caso de las empresas privadas. Con respecto a la distinción entre empresas con o sin participación extranjera, esperamos que las compañías con acceso a capital extranjero se encuentren menos restringidas de liquidez. Similarmente, es probable que, sobre todo a partir de la liberalización comercial, que coincide con la liberalización financiera, las empresas exportadoras se hayan beneficiado de un mejor acceso a créditos (debido a un mayor y más predecible valor neto empresarial), reduciendo el estrecho vínculo entre los fondos internos y la inversión.

Además del flujo de caja en interacción con dummies para cada clase de empresas de acuerdo a su tamaño, también se incluyen estas mismas variables en interacción con dummies para cada año a partir de 1989, para observar el efecto de la liberalización financiera. Cabe destacar que aquí se pueden confundir varios aspectos, debido a que además de los cambios descritos en el sistema financiero, durante el mismo período el problema de la deuda externa disminuyó en importancia, y se experimentó un gran flujo de capitales hacia el país. Como se mencionó anteriormente, durante estos años también se realizó una importante liberalización comercial, reduciéndose las barreras a la importación, y facilitando el

\footnotetext{
${ }^{22}$ Véase Gelos (1997) y Gelos (1998) para una discusión más detallada de los problemas econométricos relevantes. Allí también se presentan resultados similares a los proporcionados aquí.
} 
acceso a mercados de exportación. Probablemente estos dos cambios también hayan contribuido a aliviar las restricciones de liquidez.

Antes de discutir los resultados, es preciso señalar que existen razones por las cuales los coeficientes del flujo de caja probablemente estén sesgados hacia cero. En primer lugar la variable de flujo de caja está construida en base a las utilidades repartidas reportadas por las empresas. Por lo tanto, en los casos (probablemente aislados) en los cuales el establecimiento analizado forme parte de una empresa mayor, aumentos en las utilidades a nivel de la compañía no tienen por qué estar reflejados en mayores inversiones en cada establecimiento perteneciente a dicha empresa, ya que la compañía puede tener oportunidades de inversión más atractivas en otras de sus plantas. Además, las empresas tienen el incentivo a sobrestimar gastos, incluyendo inversiones, y subestimar ingresos. En segundo lugar, una razón más importante para sospechar que los coeficientes estarán sesgados hacia cero es que, a pesar de que la ley mexicana requiere que se reparta un diez por ciento de las utilidades de la empresa al personal ocupado, en casos especiales, la empresa puede desviarse de esa regla. Este caso es más factible cuando la empresa planea reinvertir las utilidades. Obviamente, en esta situación, se borrará toda la relación entre la variable de flujo de caja utilizada en este trabajo y la inversión. En el caso en el cual la empresa haya registrado pérdidas, las utilidades repartidas serán cero, y no negativas; sin embargo estos casos son muy aislados en la muestra utilizada. 
Cuadro 3: Modelo de Acelerador, Mínimos Cuadrados Ord. con Efectos Fijos

\begin{tabular}{|c|c|c|c|c|c|c|c|c|}
\hline & Todas & $\begin{array}{l}\text { Solo } \\
\text { priv. }\end{array}$ & $\begin{array}{l}\text { Solo con } \\
\text { part. } \\
\text { publ. }\end{array}$ & Solo Nac. & $\begin{array}{l}\text { Solo con } \\
\text { part. } \\
\text { extranj. }\end{array}$ & $\begin{array}{l}\text { Solo } \\
\text { no-exp. }\end{array}$ & Solo export. & $\begin{array}{l}\text { Solo con } \\
\text { Inv>0 }\end{array}$ \\
\hline $\begin{array}{l}\frac{C F_{i t}}{K_{i t-1}} \\
\text { (peq.) }\end{array}$ & $\begin{array}{l}0.09 \\
(6.95)\end{array}$ & $\begin{array}{l}0.09 \\
(6.50)\end{array}$ & $\begin{array}{l}0.22 \\
(3.15)\end{array}$ & $\begin{array}{l}0.08 \\
(5.24)\end{array}$ & $\begin{array}{l}0.15 \\
(5.22)\end{array}$ & $\begin{array}{l}0.04 \\
(2.32)\end{array}$ & $\begin{array}{l}0.12 \\
(2.09)\end{array}$ & $\begin{array}{l}0.07 \\
(4.72)\end{array}$ \\
\hline $\begin{array}{l}\frac{C F_{i t}}{K_{i t-1}} \\
\text { (med) }\end{array}$ & $\begin{array}{l}0.06 \\
(5.36)\end{array}$ & $\begin{array}{l}0.06 \\
(5.35)\end{array}$ & $\begin{array}{l}-0.02 \\
(-0.23)\end{array}$ & $\begin{array}{l}0.05 \\
(3.30)\end{array}$ & $\begin{array}{l}0.08 \\
(4.97)\end{array}$ & $\begin{array}{l}0.04 \\
(2.96)\end{array}$ & $\begin{array}{l}0.05 \\
(2.61)\end{array}$ & $\begin{array}{l}0.05 \\
(4.24)\end{array}$ \\
\hline $\begin{array}{l}\frac{C F_{i t}}{K_{i t-1}} \\
\text { (grandes) }\end{array}$ & $\begin{array}{l}0.09 \\
(4.25)\end{array}$ & $\begin{array}{l}0.09 \\
(4.25)\end{array}$ & $\begin{array}{l}0.06 \\
(0.45)\end{array}$ & $\begin{array}{l}0.10 \\
(4.06)\end{array}$ & $\begin{array}{l}0.05 \\
(1.77)\end{array}$ & $\begin{array}{l}0.10 \\
(3.14)\end{array}$ & $\begin{array}{l}0.10 \\
(3.15)\end{array}$ & $\begin{array}{l}0.07 \\
(3.30)\end{array}$ \\
\hline $\begin{array}{l}\frac{C F_{i t}}{K_{i t-1}} \\
\text { (después } \\
\text { pequ.) }\end{array}$ & $\begin{array}{l}-0.04 \\
(-2.21)\end{array}$ & $\begin{array}{l}-0.03 \\
(-1.97)\end{array}$ & $\begin{array}{l}-0.12 \\
(-1.45)\end{array}$ & $\begin{array}{l}-0.03 \\
(-1.81)\end{array}$ & $\begin{array}{l}-0.03 \\
(-0.80)\end{array}$ & $\begin{array}{l}0.01 \\
(0.06)\end{array}$ & $\begin{array}{l}-0.15 \\
(-2.09)\end{array}$ & $\begin{array}{l}-0.04 \\
(2.18)\end{array}$ \\
\hline $\begin{array}{l}\frac{C F_{i t}}{K_{i t-1}} \\
\text { (después } \\
\text { med.) }\end{array}$ & $\begin{array}{l}-0.00 \\
(-0.28)\end{array}$ & $\begin{array}{l}-0.00 \\
(-0.27)\end{array}$ & $\begin{array}{l}-0.01 \\
(-0.09)\end{array}$ & $\begin{array}{l}0.02 \\
(1.00)\end{array}$ & $\begin{array}{l}-0.04 \\
(-2.24)\end{array}$ & $\begin{array}{l}0.01 \\
(0.85)\end{array}$ & $\begin{array}{l}-0.01 \\
(-0.29)\end{array}$ & $\begin{array}{l}-0.01 \\
(-0.50)\end{array}$ \\
\hline $\begin{array}{l}\frac{C F_{i t}}{K_{i t-1}} \\
\text { (después } \\
\text { gr.) }\end{array}$ & $\begin{array}{l}-0.01 \\
(-0.28)\end{array}$ & $\begin{array}{l}-0.00 \\
(-0.21)\end{array}$ & $\begin{array}{l}-0.01 \\
(-0.11)\end{array}$ & $\begin{array}{l}0.01 \\
(0.25)\end{array}$ & $\begin{array}{l}-0.02 \\
(-0.06)\end{array}$ & $\begin{array}{l}-0.06 \\
(-1.56)\end{array}$ & $\begin{array}{l}-0.06 \\
(-1.56)\end{array}$ & $\begin{array}{l}0.00 \\
(0.15)\end{array}$ \\
\hline$\frac{\Delta y_{i t}}{K_{i t-1}}$ & $\begin{array}{l}0.01 \\
(4.08)\end{array}$ & $\begin{array}{l}0.01 \\
(4.08)\end{array}$ & $\begin{array}{l}0.00 \\
(-0.05)\end{array}$ & $\begin{array}{l}0.01 \\
(3.41)\end{array}$ & $\begin{array}{l}0.01 \\
(2.48)\end{array}$ & $\begin{array}{l}0.01 \\
(3.49)\end{array}$ & $\begin{array}{l}0.01 \\
(1.94)\end{array}$ & $\begin{array}{l}0.004 \\
(2.73)\end{array}$ \\
\hline $\begin{array}{l}\text { F-Stat } \\
\mathbf{R}^{2} \\
\text { No. de obs. }\end{array}$ & $\begin{array}{l}16.7 \\
0.03 \\
8340\end{array}$ & $\begin{array}{l}16.16 \\
0.03 \\
8083\end{array}$ & $\begin{array}{l}1.29 \\
0.09 \\
257\end{array}$ & $\begin{array}{l}12.0 \\
0.03 \\
6320\end{array}$ & $\begin{array}{l}6.5 \\
0.05 \\
202\end{array}$ & $\begin{array}{l}7.8 \\
0.02 \\
6022 \\
\end{array}$ & $\begin{array}{l}3.4 \\
0.04 \\
2318 \\
\end{array}$ & $\begin{array}{l}14.4 \\
0.04 \\
6779 \\
\end{array}$ \\
\hline
\end{tabular}

Nota: Nota: Variable dependiente: $I_{i t} / K_{i t-1}$. Estadístico t entre paréntesis. Para todos los años se incluyen variables dummies pero los coeficientes se omiten.

En el cuadro 3 se presentan los resultados de estas estimaciones. En las diferentes columnas se reportan los resultados de las regresiones para todas las plantas, las plantas privadas, las plantas con participación pública, las plantas nacionales, las plantas con participación extranjera, las plantas no exportadoras, las plantas exportadoras y finalmente aquellas plantas con inversión positiva. En las primeras tres líneas se presentan los coeficientes asociados al flujo de caja para cada grupo de plantas dividiendo en plantas pequeñas, medianas y grandes, para toda la muestra y de las líneas 4 a la 6 se presentan los resultados para aquella muestra que comienza después de la liberalización financiera. Finalmente, se reporta el coeficiente asociado al crecimiento del producto (efecto acelerador).

Como se puede apreciar en la primera columna, los coeficientes del flujo de caja (CF) son significativos en todos los casos excepto para el caso de las empresas públicas medianas y grandes. La magnitud del coeficiente desciende para las empresas medianas con respecto a las empresas pequeñas, como se esperaba. Sorprendentemente los coeficientes para las compañías más grandes son mayores 
que para las medianas. Solamente para las empresas pequeñas se puede observar una disminución del coeficiente después de la liberalización financiera. Aún después de la liberalización, todas las categorías de empresas parecen seguir enfrentando restricciones de liquidez.

El coeficiente del cambio en el nivel de producción tiene el signo esperado y es significativo.

Los coeficientes del flujo de caja (CF) son más bajos que los generalmente encontrados en la literatura, pero comparables a los obtenidos por Harris et al. (1994) en sus estimaciones con efectos fijos para países con un grado de desarrollo similar a México ${ }^{23}$. Además de las razones mencionadas anteriormente, los factores relacionados al ciclo de vida de las empresas pueden contribuir a este resultado, cuando se incluyen establecimientos más pequeños. Esto se debe a que las empresas que están en un proceso de crecimiento, inicialmente invierten más que el promedio de las compañías, teniendo al mismo tiempo elevados gastos y un flujo de caja relativamente bajo. Esto contribuye a disminuir la relación observada entre la inversión y las ganancias. En efecto, al analizar los gastos empresariales (la muestra contiene una lista muy completa), se puede observar una relación positiva entre las inversiones y gastos como sueldos, fletes, energía, etc. Por otra parte, las empresas que inicialmente tienen un crecimiento elevado de ventas, son las que en esos años tienden a invertir más y tener un flujo de caja menor al promedio. En los años posteriores, esta situación se revierte y las mismas empresas reducen su inversión y gozan de las utilidades proporcionadas por sus altas inversiones iniciales.

Comparando la segunda columna del cuadro 3 con la primera, no se detectan diferencias importantes. Esto se debe a que el número de empresas en la muestra con participación estatal es relativamente bajo. En cambio, la tercera columna revela diferencias importantes entre las empresas (para) estatales y las demás, ya que el coeficiente del flujo de caja (CF) es muy alto para las empresas pequeñas antes de la liberalización financiera, y los demás coeficientes no son significativos. Nótese que esto también se aplica al cambio en la producción. De estos resultados se concluye que las empresas con participación pública, excepto quizás las pequeñas, operan en un entorno muy diferente. Las empresas pequeñas parecen haberse beneficiado con respecto a su acceso al crédito después de 1989, aunque el efecto no es significativo al diez por ciento de confianza.

En el caso de las compañías con participación extranjera vs. las netamente nacionales, nuestro razonamiento ex-ante se confirma para las empresas grandes, y para las medianas después de la liberalización. Las compañías mayores con

\footnotetext{
${ }^{23}$ Robles Tapis (1987) obtiene coeficientes similares en regresiones parecidas, usando una muestra de 20 empresas durante el período 1975-84.
} 
participación extranjera no parecen haber sufrido restricciones de liquidez (el coeficiente es significativo al diez pero no al cinco por ciento) ni antes ni después del inicio de la liberalización financiera. Solamente para las empresas medianas, la situación cambia después de 1989. A diferencia de las compañías grandes, probablemente multinacionales bien establecidas en los mercados financieros internacionales, los co-propietarios extranjeros de las empresas pequeñas quizás hayan sido reticentes a fondear en el exterior sus inversiones locales en tiempos de inestabilidad macroeconómica ${ }^{24}$.

Comparando las empresas exportadoras con las no-exportadoras, se puede apreciar que se valida la predicción con respecto a las diferencias después de 1989 para las empresas pequeñas. Es interesante destacar que, de todas las empresas, las que más se vieron favorecidas después de 1989 fueron las pequeñas empresas exportadoras. Cabe notar que en una encuesta realizada para el Banco Mundial, las empresas exportadoras eran las que señalaban más frecuentemente a las altas tasas de interés como factores importantes que limitaban su crecimiento, debido a que debían competir directamente con compañías extranjeras con acceso a crédito en mejores condiciones ${ }^{25}$.

Una objeción válida a la estimación con efectos fijos concierne la posibilidad de que las variables independientes sean por los menos parcialmente endógenas. El crecimiento de la producción, por ejemplo, puede estar correlacionado con choques a la inversión a través de la función de producción ${ }^{26}$. Una solución a este tipo de problemas es la estimación con instrumentos con el método generalizado de momentos. Este método, propuesto por Arellano y Bond (1991) consiste en diferenciar las ecuaciones para eliminar el efecto fijo, y utilizar los niveles desfasados de las variables como instrumentos. El problema es que al diferenciar las variables, se tiende a aumentar los errores de medición. Además, los valores rezagados no siempre son buenos instrumentos, lo cual disminuye la precisión de la estimación. De todos modos presentamos los resultados de la estimación con el método generalizado de momentos ${ }^{27}$. La estimación se realizó en dos etapas, para corregir posibles problemas de heterocedasticidad.

\footnotetext{
${ }^{24}$ Obsérvese que los resultados Babatz y Conesa (1997) también contradicen parcialmente a las predicciones.

${ }^{25}$ The World Bank (1994).

${ }^{26}$ Véase Bond et al. (1997)

${ }^{27}$ El programa DPD de Arellano y Bond (1988), amablemente proporcionado por M. Arellano, se utilizó en la estimación.
} 
Cuadro 4: Modelo de acelerador, Efectos Fijos

Método Generalizado de Momentos (MGM)

\begin{tabular}{|c|c|c|c|c|c|c|c|c|}
\hline & Todas & $\begin{array}{l}\text { Solo } \\
\text { priv. }\end{array}$ & $\begin{array}{l}\text { Solo con } \\
\text { part. } \\
\text { publ. }\end{array}$ & Solo Nac. & $\begin{array}{l}\text { Solo con } \\
\text { part. } \\
\text { extranj. }\end{array}$ & $\begin{array}{l}\text { Solo } \\
\text { no-exp. }\end{array}$ & Solo export. & $\begin{array}{l}\text { Solo con } \\
\text { Inv }>0\end{array}$ \\
\hline$C F_{i t}$ & 0.06 & 0.06 & 0.20 & 0.07 & 0.15 & 0.06 & 0.17 & 0.06 \\
\hline$\overline{K_{i t-1}}$ & $(3.21)$ & (3.07) & (3.32) & (3.44) & $(7.35)$ & $(3.21)$ & $(4.80)$ & $(2.45)$ \\
\hline (peq.) & & & & & & & & \\
\hline$C F_{i t}$ & 0.03 & 0.03 & -0.04 & 0.01 & 0.07 & 0.04 & 0.01 & 0.02 \\
\hline$\overline{K_{i t-1}}$ & $(2.40)$ & $(2.43)$ & $(-0.36)$ & $(0.85)$ & $(5.63)$ & $(2.41)$ & $(1.80)$ & $(1.21)$ \\
\hline (med) & & & & & & & & \\
\hline$C F_{i t}$ & 0.02 & 0.02 & -0.07 & 0.03 & 0.02 & 0.01 & 0.09 & 0.03 \\
\hline$\overline{K_{i t-1}}$ & (1.29) & $(1.35)$ & $(-0.37)$ & (1.39) & (1.12) & $(0.50)$ & $(5.91)$ & $(1.52)$ \\
\hline $\begin{array}{l}\text { (grandes) } \\
C F\end{array}$ & 01 & 001 & & & & & 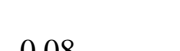 & \\
\hline & 0.01 & 0.01 & -0.09 & 0.01 & -0.09 & 0.02 & -0.08 & -0.01 \\
\hline $\begin{array}{l}K_{i t-1} \\
\text { (después }\end{array}$ & $(0.47)$ & $(0.46)$ & & $(0.29)$ & $(-3.98)$ & $(0.69)$ & $(-3.24)$ & $(-0.40)$ \\
\hline pequ.) & 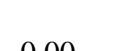 & & & & & & & \\
\hline$\frac{C_{i t}}{K}$ & $\begin{array}{l}-0.00 \\
(000)\end{array}$ & 0.00 & -0.09 & -0.01 & -0.01 & -0.00 & -0.00 & 0.03 \\
\hline $\begin{array}{l}\Lambda_{i t-1} \\
\text { (después }\end{array}$ & $(-0.09)$ & & & $(-0.44)$ & $(-0.00)$ & $(-0.03)$ & $(-0.41)$ & \\
\hline med.) & & & & & & & & \\
\hline$C F_{i t}$ & -0.04 & -0.03 & -0.01 & -0.07 & -0.02 & -0.03 & -0.06 & -0.02 \\
\hline$\overline{K_{i t-1}}$ & $(-1.98)$ & $(-1.87)$ & $(-0.06)$ & $(-3.45)$ & $(-1.04)$ & $(-1.75)$ & $(-3.55)$ & $(0.145)$ \\
\hline $\begin{array}{l}\text { (después } \\
\text { gr.) }\end{array}$ & & & & & & & & \\
\hline$\Delta y_{i t}$ & 0.01 & 0.01 & -0.02 & -0.00 & 0.003 & 0.00 & 0.00 & 0.001 \\
\hline$\overline{K_{i t-1}}$ & $(0.86)$ & $(4.08)$ & $(-1.90)$ & $(-0.11)$ & $(2.00)$ & (1.13) & $(0.69)$ & $(0.76)$ \\
\hline Test de & $2.0(2)$ & & $0.9(2)$ & $2.1(2)$ & $16.0(2)$ & $0.83(2)$ & $50.6(2)$ & $2.41(2)$ \\
\hline $\begin{array}{l}\text { Wald } \\
\text { m1 }\end{array}$ & -11.01 & -10.78 & -5.234 & -9.82 & -5.23 & -9.84 & -4.74 & -10.12 \\
\hline & -1.29 & -1.43 & 0.726 & -1.25 & -0.315 & -1.13 & -0.94 & -1.30 \\
\hline Test Sargan & $66.4(61)$ & $66.8(61)$ & $40.9(29)$ & $61.2(61)$ & $67.1(61)$ & $58(61)$ & $66(61)$ & $66.1(61)$ \\
\hline No. de obs. & 7506 & 7256 & 221 & 5688 & 1818 & 5986 & 1417 & 5945 \\
\hline
\end{tabular}

Nota: Variable dependiente: $I_{i t} / K_{i t-1}$. Estadísticas de t entre paréntesis. Dummies incluidos para todos los años (coeficientes omitidos). El modelo fue estimado en primeras diferencias, utilizando valores rezagados un año y más como instrumentos. El test de Wald es un test de significancia de todas las variables incluidas (excepto las dummies), y su distribución es de $\chi(p)$, donde p es la diferencia entre el número de instrumentos y de regresores. $\mathrm{m} 1 \mathrm{y} \mathrm{m} 2$ son tests de autocorrelación de primer y segundo orden con distribución $\mathrm{N}(0,1)$. El test de Sargan es un test de las restricciones de sobreidentificación (véase Sargan (1988)). La estimación fue realizada en dos etapas, para corregir posible heterocedasticidad.

Los resultados para las plantas pequeñas antes de la liberalización son muy similares a los del cuadro 3. Sin embargo, existen diferencias en las estimaciones de los efectos de la liberalización financiera. Salvo para las empresas pequeñas exportadoras o con participación extranjera, para las cuales se registra una reducción significativa del coeficiente sobre el flujo de caja en el período post-1989, para las demás empresas pequeñas, no se observa un mayor acceso a fondos prestables. En este contexto hay que recordar la existencia del mencionado mercado de crédito interempresarial previo a la liberalización, cuya magnitud es difícil de cuantificar. Por otra parte cabe subrayar que los dos métodos de estimación señalan que las pequeñas empresas exportadoras fueron las más favorecidas después de 1989. 
El coeficiente del flujo de caja es menor para las empresas de tamaño medio, lo cual coincide con los resultados previos y con nuestra predicción. El valor de este coeficiente para las empresas medianas no cambia significativamente después de 1989. En contraste con los resultados anteriores, las estimaciones no indican la presencia de restricciones de liquidez para las empresas grandes, con la excepción de las exportadoras antes de la liberalización. Los resultados son consistentes con una encuesta realizada entre las manufacturas eléctricas publicada en 1991, en donde la microindustria y la industria pequeña indican el difícil acceso a financiamiento como uno de los principales factores limitantes de su producción, mientras que esto no se menciona en el caso de las industrias grandes ${ }^{28}$.

Obviamente, se puede objetar que no hemos realizado un control econométrico satisfactorio con respecto a la rentabilidad futura de las empresas. Las diferencias observadas en la importancia del flujo de caja sobre las inversiones pueden deberse a diferencias en las oportunidades de inversión entre los tipos de empresas. En este respecto es interesante observar que los resultados no cambian al incluir en las regresiones la rentabilidad futura realizada. Intentos de construir una proyección de la q de Tobin usando un método similar al utilizado por Gilchrist y Himmelberg (1995) no fueron exitosos debido a problemas de multicolinearidad. Además es dudoso que en un entorno económico tan inestable, con muchos eventos discretos, una proyección lineal capture verdaderamente las expectativas empresariales. Con respecto a la q de Tobin, en varios estudios se ha observado que la omisión del q de Tobin no empeora significativamente el poder predictivo de ecuaciones de regresión de este tipo ${ }^{29}$.

Finalmente, hay que mencionar un problema que ha sido ignorado en la literatura empírica, el cual se discutirá en más detalle en la próxima sección: una fracción importante de nuestras observaciones contiene tasas de inversión de cero o ligeramente negativas. Esto requiere de atención especial al momento de la estimación. Es muy probable, por ejemplo en el caso de que existan costos fijos de inversión o que exista racionamiento de crédito, que la decisión cualitativa de invertir o no invertir se tendría que diferenciar de la decisión acerca del volumen de la inversión.

\section{IV.3 La importancia del colateral y la decisión de invertir o no invertir}

Hasta ahora, nos hemos restringido a presentar los resultados de la metodología estándar utilizada para estudiar la relación entre la inversión y el flujo de caja. Se pueden destacar dos puntos adicionales que merecen atención: el primero, mencionado al final de la sección anterior, se refiere al tratamiento de las

\footnotetext{
${ }^{28}$ CANAME (1991), p. 50. La clasificación acorde al tamaño no se explica en el texto.

${ }^{29}$ Véase Fazzari, Hubbard y Peterson (1988).
} 
observaciones con cero inversión. Este fenómeno se podría deber al racionamiento de crédito o a la existencia de costos fijos en el proceso de inversión, lo cual generalmente no se considera en la literatura. El segundo es que la teoría de las asimetrías de información en los mercados financieros no solamente pone de relieve la relación entre los fondos propios y la inversión, sino que también subraya la importancia de las garantías que la empresa pueda proveer. El objetivo de esta sección es integrar estos dos aspectos en el análisis econométrico.

La base de datos no ofrece una lista de variables financieras que permita estudiar en detalle estos aspectos. Pero por otro lado, contamos con una desagregación inusual de los activos fijos empresariales. Específicamente, podemos analizar el papel especial que juegan los bienes raíces como activos preferidos por las instituciones crediticias ${ }^{30}$.

Por otra parte las observaciones con inversión igual a cero, pueden estar indicando la presencia de costos fijos de la inversión. Un modelo muy sencillo desarrollado por Holmstrom (1993) combina los dos aspectos mencionados y sirve para aclarar estas ideas ${ }^{31}$. Allí, se describe una situación en la cual una empresa tiene una oportunidad de inversión de magnitud fija y para ello puede utilizar dos tecnologías alternativas: una eficiente y otra ineficiente. Con la tecnología eficiente, el valor presente neto esperado de la inversión es positivo, mientras que con la tecnología ineficiente, el valor presente neto es negativo. El acreedor no puede observar la tecnología elegida. Por ello, y debido al supuesto de responsabilidad limitada (el acreedor a lo sumo puede obtener el valor total del colateral) el contrato crediticio tiene que estructurarse de manera tal que se le proporcione a la empresa una renta suficiente para que tenga incentivos a escoger la tecnología eficiente. Esto, por su parte, implica que el valor neto esperado del proyecto tenga que ser estrictamente positivo. Debido a esta restricción, si el valor del colateral está por debajo de un cierto nivel, la empresa no tendrá acceso a crédito. El modelo se puede modificar de tal forma que exista un nivel mínimo de la inversión, pero que más allá de éste, la magnitud de la inversión sea variable. En este caso, la dimensión de la inversión está dictada por el volumen de colateral de la empresa.

Al no contar con datos acerca de la deuda empresarial (la base de datos solamente contiene información acerca de los pagos de intereses, y debido a los argumentos desarrollados a lo largo de este trabajo sería poco sensato asumir una misma tasa de interés para todas las empresas), nos concentraremos en los bienes raíces propiedad de la empresa. Los bienes raíces constituyen la forma de garantía

\footnotetext{
${ }^{30}$ En un contexto similar, Black, de Meza y Jeffreys (1992) encuentran una relación importante entre los precios de la vivienda y el número de fundaciones de nuevas empresas. Kiyotaki y Moore (1997) también subrayan el rol especial de los bienes raíces como garantías.

${ }^{31}$ Para una discusión más completa del modelo, véase Gelos (1997).
} 
más usual en los contratos crediticios en México ya que en raros casos se aceptan inventarios o maquinarias como colateral.

De acuerdo con el modelo discutido, esperaríamos que, ceteris paribus, la probabilidad de invertir aumente con el valor de los bienes raíces que la empresa posee y que la magnitud de la inversión también dependa del valor de las garantías. Por ello estimamos un modelo Tobit con efectos fijos, el cual se basa en la siguiente estructura:

$$
\begin{aligned}
& {\frac{I_{i t}}{K_{i t-1}}}^{*}=\beta \frac{\Delta y_{i t}}{K_{i t-1}}+\phi \frac{C F_{i t}}{K_{i t-1}}+\theta \frac{B R_{i t}}{K_{i t-1}}+\lambda_{t}+v_{i}+\varepsilon_{i t}, \\
& \frac{I_{i t}}{K_{i t-1}}={\frac{I_{i t}}{K_{i t-1}}}^{*} \text { si } \frac{I_{i t}^{*}}{K_{i t-1}^{*}}>0 \\
& =0 \text { si } \frac{I_{i t}{ }^{*}}{K_{i t-1}} \leq 0
\end{aligned}
$$

donde $B R_{i t}$ representa el valor de los bienes raíces de la empresa.

Nótese que la estimación de modelos Tobit con efectos fijos no es trivial, dado a que es difícil derivar la función de máxima verosimilitud ${ }^{32}$. Para esto utilizaremos el método propuesto por Honoré (1992) que explota la simetría de la distribución de la variable latente para generar condiciones de ortogonalidad. Estas condiciones de ortogonalidad, que tienen que ser válidas alrededor del valor verdadero de los parámetros a estimar, se utilizan para la estimación con el método generalizado de momentos (MGM) ${ }^{33}$.

Al estimar los parámetros, permitimos que los coeficientes del efecto del flujo de caja y de los bienes raíces sean diferentes antes y después del inicio de la liberalización financiera y realizamos estimaciones separadas por grupos de empresas. Los resultados pueden apreciarse en el cuadro 4. Nótese que la definición de la inversión en este caso excluye inversiones en bienes raíces, mientras que el flujo de caja incluye los flujos provenientes de ventas netas de terrenos y edificios $^{34}$.

\footnotetext{
${ }^{32}$ Véase Baltagi (1995), p. 179.

${ }^{33}$ Heckman y MaCurdy (1980) utilizan otro método para estimar un modelo Tobit con efectos fijos.

${ }^{34}$ Hay que agregar que convertimos al valor cero las pocas observaciones con inversión negativa.
} 


\section{Cuadro 5: Modelo Tobit con efectos fijos}

\begin{tabular}{|c|c|c|c|c|c|c|c|c|c|c|}
\hline & Todas & $\begin{array}{l}\text { Solo } \\
\text { priv. }\end{array}$ & $\begin{array}{l}\text { Solo con } \\
\text { part. } \\
\text { publ. }\end{array}$ & $\begin{array}{l}\text { Solo } \\
\text { Nac. }\end{array}$ & $\begin{array}{l}\text { Solo con } \\
\text { part. } \\
\text { extranj. }\end{array}$ & $\begin{array}{l}\text { Solo } \\
\text { no-exp. }\end{array}$ & $\begin{array}{l}\text { Solo } \\
\text { export. }\end{array}$ & $\begin{array}{l}\text { Solo } \\
\text { pequ. }\end{array}$ & $\begin{array}{l}\text { Solo } \\
\text { med. }\end{array}$ & $\begin{array}{l}\text { Solo } \\
\text { grandes }\end{array}$ \\
\hline$\frac{B R_{i t}}{K_{i t-1}}$ & $\begin{array}{l}0.45 \\
(5.03)\end{array}$ & $\begin{array}{l}0.44 \\
(4.73)\end{array}$ & $\begin{array}{l}0.21 \\
(0.60)\end{array}$ & \begin{tabular}{|l|}
0.46 \\
$(3.71)$
\end{tabular} & \begin{tabular}{|l|}
0.39 \\
$(3.42)$
\end{tabular} & $\begin{array}{l}0.39 \\
(3.70)\end{array}$ & $\begin{array}{l}0.51 \\
(2.37)\end{array}$ & $\begin{array}{l}0.32 \\
(1.66)\end{array}$ & $\begin{array}{l}0.52 \\
(4.34)\end{array}$ & $\begin{array}{l}0.74 \\
(3.87)\end{array}$ \\
\hline$\frac{C F_{i t}}{K_{i t-1}}$ & $\begin{array}{l}0.10 \\
(5.76)\end{array}$ & $\begin{array}{l}0.10 \\
(5.62)\end{array}$ & $\begin{array}{l}0.14 \\
(1.3)\end{array}$ & $\begin{array}{l}0.10 \\
(4.52)\end{array}$ & $\begin{array}{l}0.09 \\
(3.40)\end{array}$ & $\begin{array}{l}0.07 \\
(3.59)\end{array}$ & $\begin{array}{l}0.07 \\
(4.06)\end{array}$ & $\begin{array}{l}0.16 \\
(3.23)\end{array}$ & $\begin{array}{l}0.06 \\
(3.06)\end{array}$ & $\begin{array}{l}0.09 \\
(4.61)\end{array}$ \\
\hline$\frac{B R_{i t}}{K_{i t}}$ & $\begin{array}{l}0.10 \\
(4.45)\end{array}$ & $\begin{array}{l}0.10 \\
(5.37)\end{array}$ & $\begin{array}{l}0.07 \\
(0.63)\end{array}$ & $\begin{array}{l}0.10 \\
(3.93)\end{array}$ & $\begin{array}{l}0.11 \\
(4.71)\end{array}$ & $\begin{array}{l}0.10 \\
(4.61)\end{array}$ & $\begin{array}{l}0.12 \\
(2.39)\end{array}$ & $\begin{array}{l}0.09 \\
(2.36)\end{array}$ & $\begin{array}{l}0.10 \\
(3.74)\end{array}$ & $\begin{array}{l}0.15 \\
(2.64)\end{array}$ \\
\hline$\frac{\text { pos 89 }}{C_{i t}}$ & $\begin{array}{l}0.00 \\
(0.25)\end{array}$ & $\begin{array}{l}0.00 \\
(0.13)\end{array}$ & $\begin{array}{l}-0.04 \\
(-0.35)\end{array}$ & $\begin{array}{l}0.02 \\
(0.98)\end{array}$ & $\begin{array}{l}-0.03 \\
(-1.49)\end{array}$ & $\begin{array}{l}0.03 \\
(1.77)\end{array}$ & $\begin{array}{l}-0.01 \\
(-0.53)\end{array}$ & $\begin{array}{l}-0.01 \\
(-0.20)\end{array}$ & $\begin{array}{l}0.00 \\
(0.11)\end{array}$ & $\begin{array}{l}0.00 \\
(0.02)\end{array}$ \\
\hline$\frac{\Delta y_{i t}}{K_{i t-1}}$ & $\begin{array}{l}0.01 \\
(3.37)\end{array}$ & $\begin{array}{l}0.01 \\
(3.77)\end{array}$ & $\begin{array}{l}0.01 \\
(0.45)\end{array}$ & $\begin{array}{l}0.01 \\
(3.34)\end{array}$ & $\begin{array}{l}0.01 \\
(1.75)\end{array}$ & $\begin{array}{l}0.10 \\
(3.22)\end{array}$ & $\begin{array}{l}0.10 \\
(1.68)\end{array}$ & $\begin{array}{l}0.01 \\
(2.38)\end{array}$ & $\begin{array}{l}0.01 \\
(2.73)\end{array}$ & $\begin{array}{l}0.00 \\
(0.20)\end{array}$ \\
\hline $\begin{array}{l}\text { Test } \chi^{2} \\
\text { No. de } \\
\text { obs. }\end{array}$ & $\begin{array}{l}160 \\
8340\end{array}$ & $\begin{array}{l}163 \\
8083\end{array}$ & & $\begin{array}{l}124 \\
6320\end{array}$ & $\begin{array}{l}77 \\
2020\end{array}$ & $\begin{array}{l}106 \\
6022\end{array}$ & $\begin{array}{l}46 \\
2318\end{array}$ & $\begin{array}{l}62 \\
2573\end{array}$ & $\begin{array}{l}80 \\
4274\end{array}$ & $\begin{array}{l}72 \\
1493\end{array}$ \\
\hline
\end{tabular}

Nota: Variable dependiente: $I_{i t} / K_{i t-1}$ (excluyendo inversión en bienes raíces). Estadísticas de t entre paréntesis. Dummies incluidos para todos los años (coeficientes omitidos). Se usó el método de Newton implementado por la rutina OPTMUM de GAUSS para la optimización. El programa PANTOB descrito por Campbell y Honoré (1992) se utilizó en la estimación.

En las primeras dos líneas del cuadro 5 se presentan los coeficientes asociados a los bienes raíces y al flujo de caja para toda la muestra y en las líneas 3 y 4 para el período de liberalización financiera (1989-1994). Se puede observar que el valor de los bienes raíces tiene un efecto decisivo sobre la inversión, por encima del flujo de caja ${ }^{35}$. La importancia de los bienes raíces aumenta después de la liberalización financiera. Esto, en cierto sentido, no es sorprendente, ya que se podría argumentar que recién después de la liberalización financiera, un gran número de empresas, anteriormente completamente excluidas del crédito, hayan tenido la posibilidad de acceder a préstamos. En muchas ocasiones, estos préstamos se habrían otorgado en base a garantías, debido a que la expansión del crédito bancario no estuvo acompañada por un aumento de la capacidad de técnica de control de los bancos de magnitud similar. En otras palabras, el modelo mencionado recién es una descripción válida de la realidad que enfrentan las empresas en muchos países después de la liberalización financiera. Esta interpretación es consistente con la observación de que el coeficiente sobre los bienes raíces es mayor mientras más grande sea la empresa. Los resultados corroboran las respuestas de la encuesta antes

\footnotetext{
${ }^{35}$ Se podría sospechar que, a pesar de no incluir la inversión en bienes raíces en nuestra variable endógena, existan problemas de correlación entre la inversión en bienes raíces y la inversión en otros activos, que resulten en la correlación observada. Al respecto, se puede afirmar que la correlación entre los dos tipos de inversión es muy baja (0.027). Además, también se realizó un ejercicio en el cual solamente se incluyó en la regresion aquellos casos en los cuales el terreno (en términos reales) no varió, y se obtuvieron resultados muy similares.
} 
mencionada $^{36}$, en la cual se mencionaron a las garantías insuficientes, junto a los altos tipos de interés, como algunos de los factores que más restringen la inversión empresarial.

Nótese que acorde con los cuadros 3 y 4, las empresas públicas no se enfrentan a las mismas restricciones, ya que ninguna de las variables incluidas es significativa. Este resultado confirma el hecho de que estas empresas cuentan con garantías explícitas o implícitas, del gobierno.

Consistentemente con los resultados previos, el flujo de caja es significativo en todos los casos excepto para las empresas públicas, y este coeficiente es mayor para las empresas pequeñas que para las medianas. El efecto del flujo de caja estimado es mayor que en las regresiones con efectos fijos que no tenían en cuenta el carácter censurado de la variable inversión. Similarmente a los resultados obtenidos por el método de mínimos cuadrados con efectos fijos, este coeficiente aumenta en el caso de las empresas grandes. En contraste con los resultados anteriores, en ningún caso se puede observar una reducción significativa del coeficiente sobre CF después de la liberalización.

La teoría prediciría que para aquellas empresas con poco colateral, el efecto del flujo de caja debería ser más importante en la determinación de la inversión. En efecto, en una regresión de la inversión con efectos fijos, en la cual además de incluir el flujo de caja y el valor de los bienes raíces, se incluyó el flujo de caja en interacción con el valor del terreno, el coeficiente de esta última variable es negativo y significativo. Sin embargo, este resultado no es robusto en las estimaciones del tipo Tobit.

\footnotetext{
${ }^{36}$ The World Bank (1994).
} 


\section{Conclusiones}

A partir de los resultados econométricos obtenidos al estimar las funciones de inversión para diferentes tipos de empresas, concluimos que los factores financieros jugaron un papel importante en la determinación de las inversiones en el período 1985-94 y que en los años anteriores la restricción financiera contribuyó a los bajos niveles de inversión de los años ochenta. Entre los resultados obtenidos destaca que las empresas pequeñas fueron las más afectadas por las restricciones de liquidez. Asímismo, varias de las estimaciones indican que las pequeñas empresas exportadoras fueron las más beneficiadas por los cambios ocurridos a partir de 1989, aunque estos resultados no son robustos. Teniendo en cuenta las observaciones con inversión igual a cero, una estimación mediante un método novedoso destaca la importancia para la inversión del valor de los bienes raíces, además del flujo de caja empresarial. Estas regresiones muestran que los bienes raíces son particularmente importantes para las empresas grandes. Después de la liberalización, este tipo de garantías no solamente no perdió sino que incluso ganó importancia. Este resultado sugiere que debido a las restricciones financieras, muchas empresas simplemente no podían acceder a cierto tipo de crédito antes de 1989. Esta situación cambió a partir de 1989, sin implicar que después de este año no existieran restricciones de financiamiento. Más bien, para aquellas empresas que ahora tuvieron la oportunidad de obtener crédito en un marco más amplio, contar con garantías se volvió más importante. La expansión del crédito por parte de los bancos no estuvo acompañada por una expansión proporcional de sus capacidades de control; por ello, muchos bancos otorgaron sobre todo crédito garantizado, aumentando de esta manera la importancia de los bienes raices en la determinación del acceso al crédito de las empresas en la industria manufacturera. 


\section{APENDICE}

Las variables utilizadas fueron construidas de la forma siguiente:

Stock de capital: La encuesta incluye una variable del stock de capital para cinco categorías de activos fijos (equipos de maquinaria, construcciones, terrenos, equipos de transporte y otros) a costo de reposición para cada año. No obstante, después de un análisis de su comportamiento y después de conversar con expertos del INEGI, optamos por no utilizar estos valores debido a las altas variaciones en las series y preferimos construir estas series mediante el uso del método de inventarios perpetuos partiendo del valor de reposición reportado para el año 1984 para cada clase de activos. Con base en ese dato construimos una serie del stock de capital usando la inversión y depreciación reportada en cada año como la suma de estas variables en cada categoría de activos. Alternativamente, también utilizamos tasas de depreciación constantes, sin que ésto alterara los resultados cualitativos. Como deflactores utilizamos los índices de precios de productor de maquinaria y de construcción, el índice de precio de terrenos en la ciudad de México, y el índice de precios al mayoreo en la ciudad de México.

Inversión: La inversión es la suma de la compra de activos nuevos y usados, de las mejoras de activos y de los activos producidos menos la venta de activos, para cada categoría de activos fijos.

Flujo de caja: Inicialmente intentamos construir el flujo de caja a nivel de establecimiento en base a los ingresos y gastos reportados. En varios casos al utilizar esta variable no captamos el flujo de caja a nivel de la compañía, y quizás más importante, no se pueden considerar ingresos de actividades financieras. Por lo tanto optamos por construir el flujo de caja basándonos en las utilidades repartidas y en la depreciación reportada. Las leyes mexicanas obligan a las empresas a repartir un diez por ciento de sus utilidades al personal ocupado. Por ello multiplicamos las utilidades repartidas por diez y le agregamos la depreciación (que en la mayor parte de los casos es la depreciación contable) para obtener el flujo de caja. Obviamente, como aludimos en la sección II, la variable así construida es problemática. En algunos casos, su valor es cero (esto es el caso solamente en el 3.8 por ciento de las observaciones incluidas), y obviamente nunca llega a ser negativa. Se empleó el índice de precios al mayoreo en la ciudad de México como deflactor.

Producción: Al valor de los productos elaborados le agregamos los ingresos por servicios de maquila, restándole los gastos por servicios del mismo tipo ${ }^{37}$. La corrección se hizo a razón de que en general, la producción de las maquiladoras se registra con aquella empresa que paga por los servicios. En algunos casos, esta

\footnotetext{
${ }^{37}$ Seguimos aquí la propuesta de Grether (1994).
} 
corrección puede no ser adecuada, debido a peculiaridades en la contabilización de los servicios de maquila. Alternativamente, hubiéramos podido eliminar todas las empresas con gastos o ingresos de servicios de maquilas, lo cual hubiera reducido significativamente el número de empresas en nuestra muestra. El valor de la producción se deflactó con el índice del precio al productor, desagregado por clasificación de actividad a nivel de cuatro dígitos.

Índices de precios: Todos los índices de precios fueron obtenidos del Banco de México.

Tipo de cambio real: Se utilizó el tipo de cambio real bilateral definido en base a los índices del precio al consumidor. Fuente: Banco de México

Eliminación de empresas con valores extremos Para descartar valores inverosímiles de las variables de nuestro interés, eliminamos aquellas empresas que reportaban un capital de cero, un personal ocupado de menos de tres personas, y las que reportaban valores de las variables $\frac{\Delta y_{i t}}{K_{i t-1}}, \frac{I_{i t}}{K_{i, t-1}}$ y $\frac{B R_{i t}}{K_{i t-1}}$ que pertenecían al $3 \%$ mayor y menor de la muestra. 


\section{REFERENCIAS.}

Arellano, M. y S. R. Bond (1988), "Dynamic Panel Data Estimation using DPD - A Guide for Users". Institute for Fiscal Studies, London.

Arellano, M. y S. R. Bond (1991), "Some Tests of Specification for Panel Data: Monte Carlo Evidence on an Application to Employment Equations". Review of Economic Studies, Vol. 58(2) No. 194, Pg 277-298.

Aspe A., Pedro (1993), "El Camino Mexicano de la Transformación Económica". Textos de Economía, Fondo de Cultura Económica, 1993, México.

Athey, M. J. y P. Laumas (1994), "Internal Funds and Corporate Investment in India”. Journal of Development Economics, December 1994, Vol. 45 No. 2, Pg. 287-303.

Babatz, G. y A. Conesa (1997), "The Effect of Financial Liberalization on the Capital Structure and Investment Decisions of Firms: Evidence from Mexican Panel Data". Mimeo.

Baltagi (1995), "Econometric Analysis of Panel Data".

Bernanke, B. S. y S. Blinder (1988), "Credit, Money and Aggregate Demand". American Economic Review, June 1988, Vol. 78 No. 2, Pg. 435-439.

Bernanke, B. S. y M. Gertler (1989), "Agency Costs, Net Worth, and Business Fluctuations”. American Economic Review, March 1989, Vol. 79, No. 1, Pg. 14-31.

Bernanke, B. S., M. Gertler y S. Gilschrist (1994). "The Financial Accelerator and the Flight to Quality”. NBER Working Paper No. 4789, Cambridge, Mass.

Black, J., D. de Meza y D. Jeffreys (1992), "House Prices, the Supply of Collateral and the Enterprise Economy". Mimeo, University of Exeter.

Bond, S, J. Elston, J. Mairesse y D. Jeffreys (1992), "Financial Factors and Investment in Belgium, France, Germany and the U.K.: A Comparison Using Company Panel Data". NBER Working Paper No. 5900.

Campbell, J. R. y B. E. Honoré (1991), "Pantob Instructions”. Mimeo.

Camara Nacional de Manufacturas Eléctricas. (1991), "Monografía Económica del Sector de Manufacturas", CANAME, 1991, México. 
Copelman, M. y A. M. Werner (1997), "El Mecanismo de la Transmisión Monetaria en México". El Trimestre Económico, Ene-Mar, 1997, Vol. 64 No. 253, Pg. 75-104.

Dailami, M. (1990), "Financial Policy and Corporate Investment in Imperfect Capital Markets: The Case of Korea". World Bank Policy, Research and External Affairs Working Paper No. 409, 1990, Washington, D.C.

Dailami, M. y M. Giugale (1991), "Reflections on Credit Policy in Developing Countries: Its Effect on Private Investment", World Bank Policy, Research and External Affairs Working Paper No. 654, 1991, Washington, D.C.

Dolgow, E. y A. Aniela. (1991), "Sistema Financiero, Ahorro e Inversión en una Economía en Vías de Desarrollo: El Caso de México, 1970-1989”. Tesis (L.E.) presentada en el Instituto Tecnológico Autónomo de México (ITAM).

Fazzari, S. M., R. G. Hubbard y B.C. Petersen (1988), "Financing Constraints and Corporate Investment". Brookings Papers on Economic Activity 1, Pg. 141-206. The Brookings Institution, Washington, D.C.

Gelos, G. (1997): "How Did Financial Liberalization Affect Investment in Mexico ? Evidence from the Mexican Manufacturing Sector", Mimeo, Yale University

Gelos, G. (1998): "Fixed Investment in the Mexican Manufacturing Sector: Adjustment Costs, Credit Constraints and the Effects of Financial Liberalization", Tésis de Doctorado, Yale University

Gertler, M. y S. Gilchrist (1994), "Monetary Policy, Business Cycles and the Behavior of Small Manufacturing Firms". NBER Working Paper No. 3892, 1994, Cambridge, Mass.

Gilchrist, S. y C. P. Himmelberg (1995), "Evidence on the Role of Cash Flow for Investment". Journal of Monetary Economics, December 1995, Vol. 36 No. 3, Pg. 541-572.

Goldberg, L. (1996), "Exchange Rates and Investment Response in Latin America". Mimeo.

Greenham L., Laura E. (1997), "Mecanismo de Transmisión de Política Monetaria en México". Tesis (L.E.) presentada en el Instituto Tecnológico Autónomo de México (ITAM). 
Gruben, W. C. y R. P. McComb (1997), "Liberalization, Privatization and Crash: Mexico's Banking System in the 1990's". Economic Review, Federal Reserve Bank of Dallas, First Quarter 1997, Pg. 21-30.

Gunther, J. W. y R. R. Moore (1993), "Credit Conditions and Macroeconomic Activity: Evidence from Mexico". Documento de Trabajo, Financial Industry Studies Department, Federal Reserve Bank of Dallas.

Grether, J. M. (1995), “Trade Liberalization, Market Structure, and Performance in Mexican Manufacturing: 1985-90”. Mimeo.

Härdle, W. (1990), “Applied Nonparametric Regression”, Cambridge University Press, Cambridge

Harris, J. R., F. Schiantarelli, M. G. Siregar (1994), "The Effect of Financial Liberalization on the Capital Structure and Investment Decsions of Indonesian Manufacturing Establishments". The World Bank Economic Review, January 1994, Vol. 8 No. 1, Pg. 17-48.

Heckman, J. J. y T. E. MaCurdy (1980), "A Life Cycle Model of Female Labour Supply”, Review of Economic Studies, 1980, Vol. 57(1) No. 146, Pg. 47-78.

Holmstrom, B. (1993), "Financing of Investment in Eastern Europe: A Theoretical Perspective". Mimeo.

Honoré, B. E., "Trimmed LAD and Least Squares Estimation of Truncated and Censored Regression Models with Fixed Effects". Econometrica, May 1992, Vol. 60, No. 3, Pg. 533-565.

Hubbard, R.G. (1998), "Capital-Market Imperfections and Investment", Journal of Economic Literature, Vol. 36, No. 1, Pg. 193-225

Jaramillo, F, F. Schiantarelli, F. y A. Weiss (1993), "Capital Market Imperfections before and after Financial Liberalization: An Euler-equation Approach to Panel Data for Ecuadorian Firms”, IBRD Working Paper No. 1091, 1993, Washington, D.C.

Kanchuger, R., F. Lysy, y D. Oaks (1994), "Mexico Country Economic Memorandum Fostering Private Sector Development in the 1990's", IBRD Staff Appraisal Report, No11823, 1994, Washington, D.C.

Kiyotaki, N. y J. Moore (1997), "Credit Cycles". Journal of Political Economy, April 1997, Vol. 105 No. 2, Pg. 211-248. 
Levine, Ross (1998), "Financial Development and Economic Growth: Views and Agenda", Journal of Economic Literature, Vol. 35, No. 2, Pg. 688-726

Mansell Carstens, C. (1995), "Las Finanzas Populares En México - El Redescubrimiento de un Sistema Financiero Olvidado". Centro de Estudios Monetarios Latinoamericanos, Editorial Milenio, ITAM, 1995, México.

McKinnon, Ronald I. (1976), "Money and Finance in Economic Growth and Development": Essays in Honor of Edward S. Shaw; proceedings of the Conference held at Stanford Univesity, Dekker, Inc., 1976, New York.

Meyer, John R. y Kuh, E. (1959), "The Investment Decision: An Empirical Study". Harvard University Press, 1959, Cambridge, Mass.

Modigliani, F. y M. Miller (1958), "The Cost of Capital Corporate Finance, and the Theory of Investment”, American Economic Review, June 1958, Vol. 48 No. 3, Pg. 261-297.

Myers, S. C. y N.S. Majluf (1984), "Corporate Financing and Investment Decisions when Firms have Information that Investors do not have". NBER Working Paper No. 1396, 1984, Cambridge, Mass.

Nabi, I. (1989), "Investment in Segmented Capital Markets”, The Quarterly Journal of Economics, August 1989, Vol. 104 No. 3, Pg. 453-462.

Rama, Martin (1990), "Empirical Investment Equations in Developing Countries". IBRD Working Paper No. 563, 1990, Washington, D.C.

Robles Tapia, G. (1987), "La Inversion Privada y el Sistema Impositivo en un Contexto Inflacionario: Evidencia Microeconométrica para México”. Tesis (L.E.) presentada en el Instituto Tecnológico Autónomo de México (ITAM).

Sargan, J. D. (1988), "Testing for Misspecification after Estimating using Instrumental Variables", en E. Maasoumi (Ed.), "Contributions to Econometrics: John Dennis Sargan”, Vol. 1, 1988, Cambridge University Press.

Shaw, E. S. (1973), "Financial Deepening in Economic Development". Oxford University Press, Economic Development Series, 1973, New York.

Schiantarelli, F. (1996), "Financial Constraints and Investment Methodological Issues and International Evidence". Oxford Review of Economic Policy, Summer 1996, Vol. 12, No. 2, Pg. 70-89. 
Skiles, M. E. (1991), "Stabilization and Financial Sector Reform in Mexico". Federal Reserve Bank of New York Research Paper No. 9125, 1991, New York.

Stiglitz, J. y A. Weiss (1981), "Credit Rationing in Markets with Imperfect Information". American Economic Review, June 1981, Vol. 71, No. 3, Pg. 393-410.

Townsend, Robert (1979), "Optimal Contracts and Competitive Markets with Costly State Verification". Journal of Economic Theory 2, October 1979, Vol. 21 No. 2, Pg. 265-293.

Tybout, J. R. (1983), "Credit Rationing and the Investment Behavior in a Developing Country". Review of Economics and Statistics, November 1983, Vol. 65 No. 4, Pg. 598-607.

Warner, A. M. (1993), "Did the Debt Crisis or Declining Oil Prices cause Mexico's Investment Collapse?”, IBRD Working Paper No. 1102, 1993, Washington, D.C. 